\title{
Instabilities in nematic elastomers in external electric and magnetic fields
}

\author{
A.M. Menzel ${ }^{1, \mathrm{a}}$ and H.R. Brand ${ }^{1,2, \mathrm{~b}}$ \\ 1 Theoretische Physik III, Universität Bayreuth, 95440 Bayreuth, Germany \\ 2 Max Planck Institute for Polymer Research, P.O. Box 3148, 55021 Mainz, Germany
}

Received 23 October 2007 and Received in final form 20 February 2008

Published online: 7 May 2008 - C EDP Sciences / Società Italiana di Fisica / Springer-Verlag 2008

\begin{abstract}
In this paper we study the macroscopic behavior of nematic side-chain liquid single crystal elastomers exposed to an external electric or magnetic field. For this purpose we use the framework of a continuum model. The geometries investigated comprise the bend and the twist geometry known from the classical Frederiks transition in low molecular weight liquid crystals. For the bend geometry we find a laterally homogeneous and a two-dimensional undulatory instability, which may compete at onset. In the case of the twist geometry three instabilities can occur at onset, two of which are two dimensional and clearly show undulations. As a major result we propose how the values of the twist coefficient $K_{2}$ and the values of the material parameters $D_{1}$ and $D_{2}$ connected to relative rotations between the director field and the polymer network can be determined from experimental observations. In addition, we explain why a twist experiment is probably the most suitable set-up in order to measure the material parameter $D_{1}$.
\end{abstract}

PACS. 61.30.Vx Polymer liquid crystals - 61.30.Dk Continuum models and theories of liquid crystal structure - 61.30.Gd Orientational order of liquid crystals; electric and magnetic field effects on order

\section{Introduction}

The synthesis of nematic side-chain liquid crystal elastomers was first reported in 1981 [1]. It can be performed by chemically crosslinking polymer chains to which mesogenic units had been attached as side groups, the so-called side-chain liquid crystalline polymers [2]. If this crosslinking occurs in the absence of an external field, polydomain structures are obtained that do not show a homogeneous orientation of the liquid crystalline director field over the whole sample. However, in the following we will confine our considerations to nematic side-chain liquid single crystal elastomers (SCLSCEs). Here, the director field is uniformly oriented over the whole sample in its ground state. These materials have to be generated by special ways of synthesis: during the final crosslinking step the mesogenic units must on average be oriented in a certain direction by the influence of an external field, which can be mechanical, electric, magnetic, or imposed by the boundaries of the system $[3,4]$. This special direction then gets locked in or "frozen in" [5], so that no spontaneous breaking of rotational symmetry occurs at the "transition" to the nematic state. Consequently, a state referred to as "paranematic", or supercritical behavior can be observed $[6,7]$.

\footnotetext{
a e-mail: andreas.menzel@uni-bayreuth.de

b e-mail: brand@uni-bayreuth.de
}

The preferred direction remains imprinted in all the different states.

It is worthwhile to investigate the behavior of SCLSCEs when exposed to an external electric or magnetic field, also in view of their potential applications which include, e.g., artificial muscles [8-10]. The geometries that we want to study in the following correspond to those suitable to observe the classical Frederiks transition in common low molecular weight liquid crystals (LMWLCs). They include the so-called geometries of splay, bend and twist [11]. As a characteristic feature of these three geometries the sample is confined between two parallel plates that have a fixed distance. The ground-state director orientation is imposed by a preferred direction at the system boundaries, which is enforced by a special treatment of the plate surfaces. An electric or magnetic field is then applied in a direction perpendicular to the ground-state orientation. For the appropriate sign of the electric or magnetic anisotropy of the sample, the director tends to align parallel to the external field direction. On the other hand, this reorientation of the director field is hindered by an energetically unfavored distortion of the director field, which is due to the imposed boundary conditions. The Frederiks transition takes place, when at a critical electric or magnetic field amplitude the director overcomes this barrier and starts to reorient.

For nematic SCLSCEs the situation is more complicated as the director is not only anchored at the system 
boundaries. Additionally, the director orientation is coupled to the polymer matrix, a fact that we will take into account by including the variables of relative rotations as further illustrated later on.

Among the three geometries mentioned, the splay geometry is the most suitable one to perform experimental observations because it is the only one where the external field is applied perpendicularly to the plate surfaces. This geometry with or without anchoring of the director orientation at the plate surfaces has for example been studied experimentally for swollen nematic SCLSCEs [12] and nematic elastomeric gels [13], theoretically making use of a mesoscopic model based on rubber elasticity [14,15], and numerically [16].

In this paper, however, we will apply a linearized macroscopic description as it was used in reference [17] in order to analyze the splay geometry. This description has the advantage that spatial variations can easily be included. Indeed, in this way a new undulatory instability has been found for nematic SCLSCEs. At onset, in the splay geometry, it competes with a Frederiks-like instability for strong anchoring boundary conditions [17]. Later on, we generalized this investigation of the splay geometry to the description of cholesteric SCLSCEs, and again we have found the competition between an undulatory and a Frederiks-like instability [18].

Here, we will mainly restrict ourselves to the description of nematic SCLSCEs confined in the bend and twist geometry. We will demonstrate that experimental investigations of these two geometries would be very important in spite of the larger challenges that result from the way of applying the external field. We will show that also for these two geometries undulatory instabilities can be found at onset, and that conclusions on the values of several material parameters can be drawn that cannot be measured directly up to now. In particular, this is on the one hand the value of the twist coefficient $K_{2}$ connected to the twist deformation of the director field, but not to any coupling of the director to the polymer network. On the other hand, these are the material parameters $D_{1}$ and $D_{2}$ associated with relative rotations between the director field and the polymer network.

The paper is organized as follows: in the next section, we shortly describe the linearized continuum model with the help of which we characterize the materials. In Section 3 we will summarize the results obtained in reference [17] for the splay geometry, and then we study the formally closely connected bend geometry. After that, in Section 4, we will investigate the twist geometry in detail. Finally, we will summarize and discuss our results in Section 5, and we will give a short perspective. Some details of the analysis are presented in several appendices.

\section{Linearized continuum model of nematic SCLSCEs}

As we have already mentioned before, we will use a linearized continuum model in order to characterize the behavior of the materials. For the case of nematic and cholesteric SCLSCEs in an external field, this model has already been described in detail elsewhere [17-20]. We therefore only summarize its basic elements.

In our picture the materials consist of two subsystems. One of them represents the observed elastic behavior of the polymer network, and the other component shows the liquid crystalline behavior emerging from the average orientation of the mesogenic side-groups. Additionally, these two subsystems are coupled to each other. Such a view of the materials is justified if the crosslinking density is not too large [21].

For this purpose, we have to identify the appropriate macroscopic variables in order to describe the materials. By symmetry arguments we can then combine them to obtain an expression for the corresponding thermodynamic potential. In our considerations, we will call this thermodynamic potential the "generalized energy". It can be viewed as one of the classical thermodynamic potentials extended in order to account for the behavior of nematic SCLSCEs in an external electric field.

Minimizing the resulting expression for the generalized energy with respect to the independent variables that correspond to the macroscopic degrees of freedom, we can determine the actual state of the system under the influence of a static external field.

The first three macroscopic variables that can contribute to the generalized energy density are the three variables already present in the hydrodynamic description of a simple liquid. These are the densities of mass $\rho$, of momentum $\boldsymbol{g}$, and of energy $\varepsilon$. Their influence will not be studied in the mainly static considerations that follow.

In order to include the elastic behavior we introduce a displacement field $\boldsymbol{u}(\boldsymbol{r})$ [22]. Shear elastic deformations and deformations of compression and dilation then enter the expression for the generalized energy density via the components of the elastic strain tensor $\varepsilon_{i j}=\frac{1}{2}\left(\partial_{i} u_{j}+\right.$ $\left.\partial_{j} u_{i}\right)$.

The local state of the nematic component within the liquid crystalline state is characterized by the director field $\hat{\boldsymbol{n}}(\boldsymbol{r})$ [11]. $\hat{\boldsymbol{n}}$ is a unit vector, where the states $+\hat{\boldsymbol{n}}$ and $-\hat{\boldsymbol{n}}$ cannot be distinguished. Only gradients of the director field $\partial_{i} n_{j}$ can contribute directly as macroscopic variables to the expression of the generalized energy density.

Finally, the coupling between the two subsystems is taken into account by the variables of relative rotations [23]. They contain the difference between a local rotation of the nematic director and a local rotation of the polymer network, and they have to be included in a hydrodynamic description of the materials as slowly relaxing variables [19]. Relative rotations are decisive in our characterization of SCLSCEs because they account for many of the outstanding properties of this class of materials (compare, e.g. [24]).

In the linear regime, relative rotations $\tilde{\Omega}$ are given by the difference between the local variations of the director field $\boldsymbol{\delta} \boldsymbol{n}(\boldsymbol{r})$ and the local rotation of the polymer network perpendicularly to the director, denoted by $\boldsymbol{\Omega}^{\perp}(\boldsymbol{r})$ :

$$
\tilde{\Omega}(\boldsymbol{r})=\boldsymbol{\delta} \boldsymbol{n}(\boldsymbol{r})-\boldsymbol{\Omega}^{\perp}(\boldsymbol{r}) .
$$


Here, $\boldsymbol{\delta} \boldsymbol{n}(\boldsymbol{r})=\hat{\boldsymbol{n}}(\boldsymbol{r})-\hat{\boldsymbol{n}}_{0}$ with $\hat{\boldsymbol{n}}_{0}$ being the ground-state director orientation. $\boldsymbol{\Omega}^{\perp}(\boldsymbol{r})$ can be parameterized by $\Omega_{i}^{\perp}=n_{j} \Omega_{j i}$, where $\Omega_{i j}=\frac{1}{2}\left(\partial_{i} u_{j}-\partial_{j} u_{i}\right)$ gives the local rotation of the polymer network. (Here and throughout the paper, summation over repeated indices is implied.) It follows that

$$
\hat{\boldsymbol{n}} \cdot \tilde{\boldsymbol{\Omega}}=0 .
$$

In the nonlinear regime the situation becomes more complicated. An extension of the variables of relative rotations to the nonlinear regime and an illustrative example for the interesting consequences can be found in [25].

We now combine these variables to derive all contributions up to quadratic order. This way, by symmetry arguments, we obtain an expression for the generalized energy density of a nematic SCLSCE in a static homogeneous external electric field $\boldsymbol{E}$ :

$$
\begin{aligned}
F= & F_{0}+c_{1} \varepsilon_{i j} \varepsilon_{i j}+\frac{1}{2} c_{2} \varepsilon_{i i} \varepsilon_{j j} \\
& +\frac{1}{2} D_{1} \tilde{\Omega}_{i} \tilde{\Omega}_{i}+D_{2} \tilde{\Omega}_{i} \varepsilon_{i j} n_{j} \\
& +\frac{1}{2} K_{1}(\nabla \cdot \hat{\boldsymbol{n}})^{2}+\frac{1}{2} K_{2}[\hat{\boldsymbol{n}} \cdot(\nabla \times \hat{\boldsymbol{n}})]^{2} \\
& +\frac{1}{2} K_{3}[\hat{\boldsymbol{n}} \times(\nabla \times \hat{\boldsymbol{n}})]^{2} \\
& -\frac{1}{2} \epsilon_{a}\left(n_{i} E_{i}\right)^{2}-e_{1}\left(n_{i} E_{i}\right)\left(\partial_{j} n_{j}\right)-e_{3} n_{i}\left(\partial_{i} n_{j}\right) E_{j} .
\end{aligned}
$$

Here, $F_{0}$ contains all the terms including variables already present in the description of a simple liquid, and it contains the energy density arising solely from the external electric field. Via the terms with the coefficients $c_{1}$ and $c_{2}$ we characterize the elastic behavior of the polymer network, which for simplicity is assumed to be isotropic [22]. After that, the terms including the variables of relative rotations and thus the coupling between the director and the polymer network are listed [23]. Up to now, no experiment has been proposed to measure the values of the corresponding material parameters $D_{1}$ and $D_{2}$ directly. To address this question will be a major issue in the following sections. Next, in expression (3), there is the Frank energy density for a nematic liquid crystalline phase [11]. Finally the contributions involving the external electric field are added [11]. $\epsilon_{a}$ denotes the dielectric anisotropy and is assumed to be positive throughout this paper, because otherwise no effect will be observed in the geometries investigated. $e_{1}$ and $e_{3}$ are the flexoelectric coefficients [11]. However, as we have shown in Appendix A, the flexoelectric terms play no role for the solutions found. We will therefore not refer to the flexoelectric contributions in the main text of this paper anymore. The situation for an external magnetic field $\boldsymbol{H}$ then becomes formally identical to the one for an external electric field, and we obtain the corresponding results simply by replacing $E_{i} \rightarrow H_{i}$ and $\epsilon_{a} \rightarrow \chi_{a}$, where $\chi_{a}$ denotes the diamagnetic anisotropy.

Electrostrictive and magnetostrictive effects have been neglected as they are known to be small. Furthermore, we will assume the material to be a perfect electric insulator throughout this paper.
The thermodynamic stability conditions arising from equation (3) read $c_{1}>0,2 c_{1}+3 c_{2}>0, D_{1}>0, D_{2}^{2}<$ $4 c_{1} D_{1}, K_{1}>0, K_{2}>0$, and $K_{3}>0[11,22,23]$.

We note that in reference [17] the material parameter $D_{2}$ was defined with the opposite sign, which becomes evident when we contrast the respective definition of $\Omega^{\perp}(\boldsymbol{r})$ (and $\boldsymbol{\delta} \boldsymbol{n}(\boldsymbol{r})$ ). This point will become important in the next section, in which we investigate a nematic SCLSCE in the bend geometry and compare the results with those obtained in reference [17] for the splay geometry.

\section{Bend geometry}

In this section we first want to briefly summarize the main results obtained in reference [17] for the splay geometry. The latter is sketched on the left of Figure 1. As will always be assumed in this paper, the sample is confined between two parallel plates such that its boundaries coincide with the planes $z=0$ and $z=d$ of our Cartesian coordinate system. The whole system is assumed to be infinitely extended in lateral directions, which is of course an approximation. The elastomer cannot penetrate the plates, and it should not detach from the plate surfaces. In other words we obtain as a boundary condition for the displacement field

$$
u_{z}(z=0) \equiv u_{z}(z=d) \equiv 0 .
$$

In the splay geometry we further impose as a boundary condition for the director field

$$
\hat{\boldsymbol{n}}(z=0) \equiv \hat{\boldsymbol{n}}(z=d) \equiv\left(\begin{array}{l}
1 \\
0
\end{array}\right) \equiv \hat{\boldsymbol{n}}_{0},
$$

and the external electric field reads $\boldsymbol{E}=E \hat{\boldsymbol{z}}$. The system can be treated as effectively two dimensional, which is also confirmed by the experiments: the director reorients within the plane containing $\hat{\boldsymbol{n}}_{0}$ and $\boldsymbol{E}$, and there is no considerable distortion observed in the direction perpendicular to this plane [12].

By means of a linear stability analysis, a critical amplitude of the external electric field can be determined. At this critical field amplitude, the ground-state director orientation becomes unstable with respect to an instability of the orientation of the director field and of the distortion of the polymer network. However, as shown in reference [17], in the splay geometry there exist two qualitatively different kinds of instabilities that compete at onset.

One of these two instabilities is laterally homogeneous (i.e. homogeneous in $x$-direction). It is the analog of the classical Frederiks instability, concerning the director reorientation. The amplitude of the latter varies like $\sin \left(\frac{\pi}{d} z\right)$, and it is illustrated in the upper part of Figure 2 on the left. The corresponding distortion of the polymer network in $x$-direction is sketched in the lower half on the left. It is also laterally homogeneous and varies like $\cos \left(\frac{\pi}{d} z\right)$. We assume that the elastomer may slip along the plate surfaces in lateral directions, which can experimentally be realized for example by lubrication with silicone oil [12]. The distortion of the elastomer in $z$-direction vanishes at onset. 

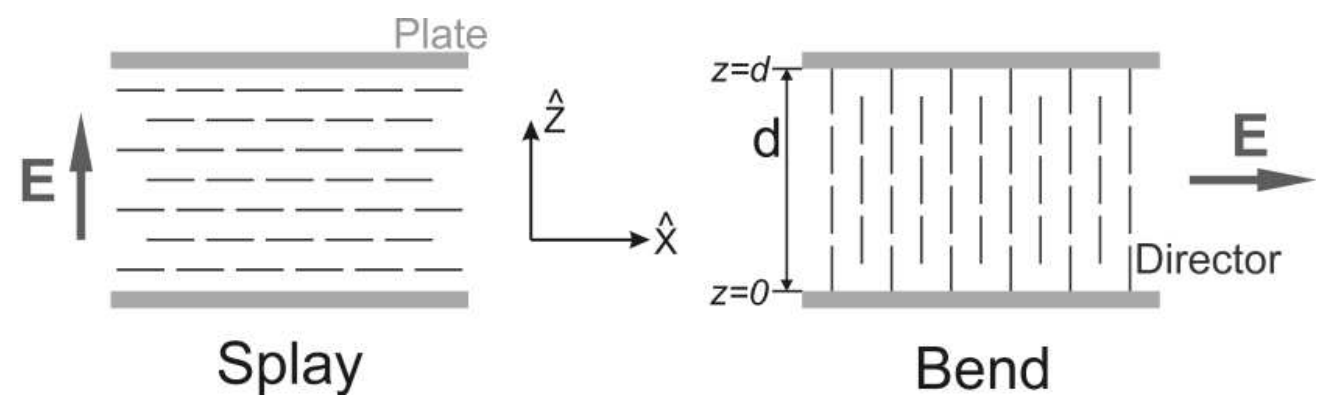

Bend

Fig. 1. Sketch of the ground-state director orientation of a nematic SCLSCE in the splay (left) and bend (right) geometry.
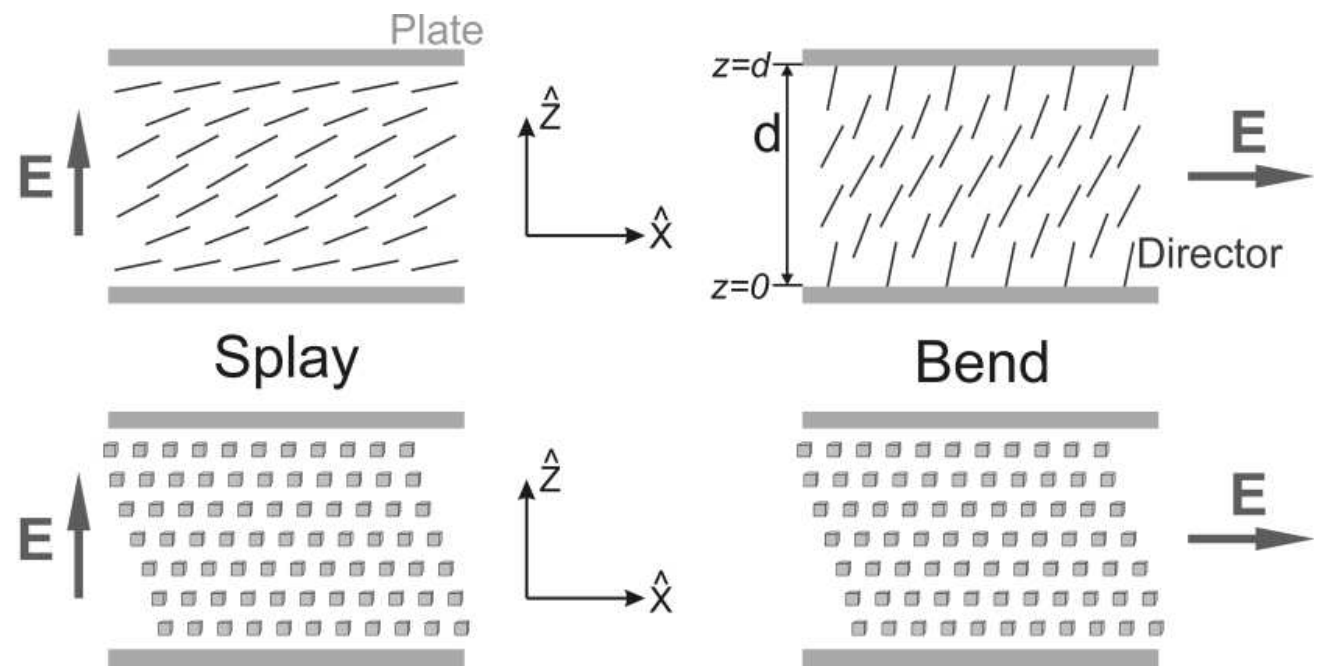

Fig. 2. Director reorientation (above) and lateral distortion of the polymer network (below) occurring during the Frederiks-like instability shown by a nematic SCLSCE in the splay (left) and bend (right) geometry.
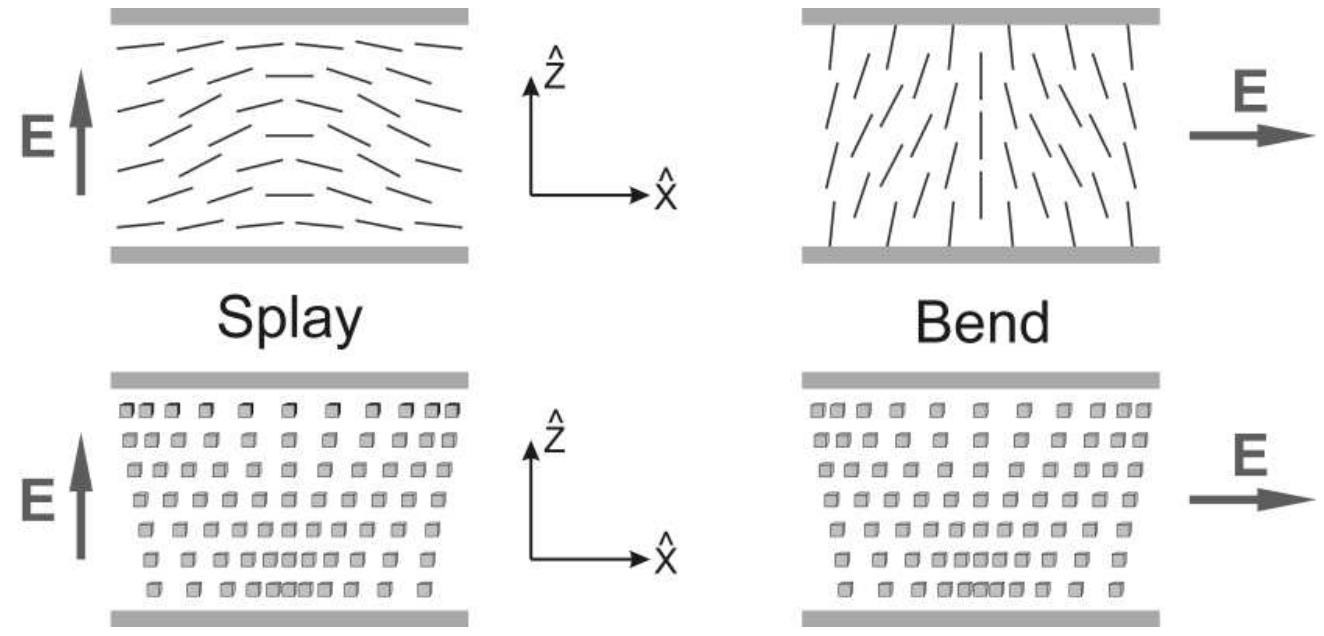

\section{Bend}

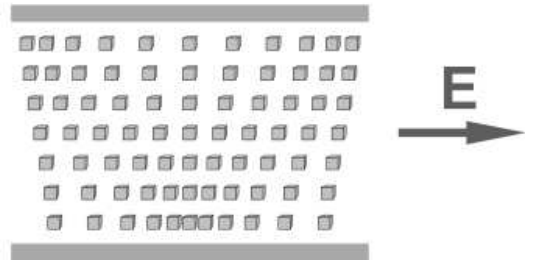

Fig. 3. Director reorientation (above) and lateral distortion of the polymer network (below) occurring during the undulatory instability shown by a nematic SCLSCE in the splay (left) and bend (right) geometry.

When the other, qualitatively different instability occurs at onset, the reorientation of the director field and the distortion of the polymer network are additionally undulated in $x$-direction. We illustrated this on the left of Figure 3. Furthermore, the distortion in $z$-direction does not vanish at onset any more, but in general it varies like $\sin \left(\frac{\pi}{d} z\right)$ with additional undulations in $x$-direction.
In reference [17] the authors investigated the two limiting cases of very short and very large wavelengths of the lateral undulations, compared to the sample thickness $d$. They studied the question, which one of the two possible instabilities will occur at onset, that is which one of them has a smaller corresponding critical field amplitude. As a result, they found that only for a negative sign of 
the material parameter $D_{2}$ (positive sign in reference [17], compare ending of Section 2) the undulatory instability will be detected at onset with certainty, if the sample thickness is large enough. In reference [18] we studied this question numerically for cholesteric SCLSCEs. There again, we only found the undulations to occur at onset for negative values of $D_{2}$, and we gave an explanation for this relation. We therefore conclude that in the limiting case of a nematic SCLSCE in the splay geometry undulations only occur at onset if $D_{2}$ is negative.

We now turn to the investigation of the bend geometry sketched on the right of Figure 1, in analogy to the study of the splay geometry in [17]. Like the splay geometry, also the bend geometry can be treated as effectively two dimensional. The homogeneous external electric field is oriented in $x$-direction, $\boldsymbol{E}=E \hat{\boldsymbol{x}}$, and we now obtain the boundary conditions

$$
\hat{\boldsymbol{n}}(z=0) \equiv \hat{\boldsymbol{n}}(z=d) \equiv\left(\begin{array}{l}
0 \\
1
\end{array}\right) \equiv \hat{\boldsymbol{n}}_{0}
$$

in addition to equation (4). As independent variables corresponding to the macroscopic degrees of freedom we identify two components $u_{x}$ and $u_{z}$ of the displacement field, as well as one component $n_{x}$ of the director field due to $\hat{\boldsymbol{n}}^{2}=1$.

Expressing the generalized energy density $F$ from equation (3) in these three variables, we obtain

$$
\begin{aligned}
F= & F_{0}+c_{1}\left\{\left(\partial_{x} u_{x}\right)^{2}+\frac{1}{2}\left[\left(\partial_{x} u_{z}\right)+\left(\partial_{z} u_{x}\right)\right]^{2}+\left(\partial_{z} u_{z}\right)^{2}\right\} \\
& +\frac{1}{2} c_{2}\left\{\left(\partial_{x} u_{x}\right)+\left(\partial_{z} u_{z}\right)\right\}^{2} \\
& +\frac{1}{2} D_{1}\left\{n_{x}^{2}+n_{x}\left[\left(\partial_{x} u_{z}\right)-\left(\partial_{z} u_{x}\right)\right]\right. \\
& \left.\quad+\frac{1}{4}\left[\left(\partial_{x} u_{z}\right)-\left(\partial_{z} u_{x}\right)\right]^{2}\right\} \\
& +\frac{1}{2} D_{2}\left\{n_{x}\left[\left(\partial_{x} u_{z}\right)+\left(\partial_{z} u_{x}\right)\right]\right. \\
& \left.+\frac{1}{2}\left(\partial_{x} u_{z}\right)^{2}-\frac{1}{2}\left(\partial_{z} u_{x}\right)^{2}\right\} \\
& -\frac{1}{2} \epsilon_{a} E^{2} n_{x}^{2} \\
& +\frac{1}{2} K_{1}\left(\partial_{x} n_{x}\right)^{2}+\frac{1}{2} K_{3}\left(\partial_{z} n_{x}\right)^{2} .
\end{aligned}
$$

In order to observe a transition in an experiment, one would slowly increase the amplitude of the external field such that the system is always in a state of thermodynamic equilibrium. In such a state, the generalized energy $\mathcal{F}=$ $\int_{V} F \mathrm{~d}^{3} r$ is minimal ( $V$ being the volume of the sample). We can thus find the actual current state of the system for a given external field amplitude by minimizing $\mathcal{F}$ with respect to the three independent variables $u_{x}, u_{z}$, and $n_{x}$. This leads us to three coupled partial differential equations that characterize the state of the bulk of the sample:

$$
\begin{aligned}
0 \equiv \frac{\delta \mathcal{F}}{\delta u_{x}} \equiv & C\left(\partial_{x}^{2} u_{x}\right)+\beta\left(\partial_{z}^{2} u_{x}\right)+\left(c_{2}+\gamma\right)\left(\partial_{x} \partial_{z} u_{z}\right) \\
& -\alpha\left(\partial_{z} n_{x}\right) \\
0 \equiv \frac{\delta \mathcal{F}}{\delta u_{z}} \equiv & C\left(\partial_{z}^{2} u_{z}\right)+\tilde{\beta}\left(\partial_{x}^{2} u_{z}\right)+\left(c_{2}+\gamma\right)\left(\partial_{x} \partial_{z} u_{x}\right) \\
& -\tilde{\alpha}\left(\partial_{x} n_{x}\right) \\
0 \equiv \frac{\delta \mathcal{F}}{\delta n_{x}} \equiv & -\tilde{\alpha}\left(\partial_{x} u_{z}\right)-\alpha\left(\partial_{z} u_{x}\right)+\left\{D_{1}-\epsilon_{a} E^{2}\right\} n_{x} \\
& -K_{1}\left(\partial_{x}^{2} n_{x}\right)-K_{3}\left(\partial_{z}^{2} n_{x}\right)
\end{aligned}
$$

In analogy to reference [17] we have introduced the abbreviations

$$
\begin{aligned}
& C=2 c_{1}+c_{2}, \\
& \alpha=\frac{1}{2} D_{1}-\frac{1}{2} D_{2}, \\
& \tilde{\alpha}=-\frac{1}{2} D_{1}-\frac{1}{2} D_{2}, \\
& \beta=c_{1}+\frac{1}{4} D_{1}-\frac{1}{2} D_{2}, \\
& \tilde{\beta}=c_{1}+\frac{1}{4} D_{1}+\frac{1}{2} D_{2}, \\
& \gamma=c_{1}-\frac{1}{4} D_{1} .
\end{aligned}
$$

In order to find the critical Fourier mode that becomes unstable first at the corresponding critical electric field amplitude $\left|E_{c}\right|$, we solve the system of equations (8)-(10) by the ansatz

$$
\begin{aligned}
& u_{x}(x, z)=A \sin \left(q_{x} x+\vartheta\right) \cos \left(q_{z} z\right), \\
& u_{z}(x, z)=B \cos \left(q_{x} x+\vartheta\right) \sin \left(q_{z} z\right), \\
& n_{x}(x, z)=N \sin \left(q_{x} x+\vartheta\right) \sin \left(q_{z} z\right) .
\end{aligned}
$$

Here, $A, B$, and $N$ denote the respective amplitudes that are considered to be constant in our linear stability analysis. $q_{x}$ and $q_{z}$ are the wave numbers by which the solution may be modulated in $x$ - and $z$-direction, and $\vartheta$ is an arbitrary phase angle (which, however, may not simply be set equal to zero without taking care of the case $q_{x}=0$ separately).

Introducing ansatz (17)-(19) into equations (8)-(10), we obtain a homogeneous linear system of equations for the amplitudes $A, B$, and $N$ :

$$
\left(\begin{array}{ccc}
C q_{x}^{2}+\beta q_{z}^{2} & \left(c_{2}+\gamma\right) q_{x} q_{z} & \alpha q_{z} \\
\left(c_{2}+\gamma\right) q_{x} q_{z} & C q_{z}^{2}+\tilde{\beta} q_{x}^{2} & \tilde{\alpha} q_{x} \\
\alpha q_{z} & \tilde{\alpha} q_{x} & M
\end{array}\right)\left(\begin{array}{c}
A \\
B \\
N
\end{array}\right)=0
$$

where $M=D_{1}-\epsilon_{a} E^{2}+K_{1} q_{x}^{2}+K_{3} q_{z}^{2}$.

We can see that the ground state $A=B=N=0$ is always a solution to this system of equations, however, at a certain critical electric field amplitude $\left|E_{c}\right|$, the ground state becomes unstable. This critical field amplitude can 
be determined by setting the determinant of the matrix from equation (20) equal to zero, which leads us to

$\epsilon_{a} E^{2}\left(q_{x}, q_{z}\right)=D_{1}+K_{1} q_{x}^{2}+K_{3} q_{z}^{2}-\frac{S_{1} q_{x}^{4}+S_{2} q_{x}^{2} q_{z}^{2}+S_{3} q_{z}^{4}}{T_{1} q_{x}^{4}+T_{2} q_{x}^{2} q_{z}^{2}+T_{3} q_{z}^{4}}$

where, according to reference [17], we have defined the abbreviations

$$
\begin{aligned}
& S_{1}=C \tilde{\alpha}^{2}, \\
& S_{2}=\tilde{\alpha}^{2} \beta+\alpha^{2} \tilde{\beta}-2 \alpha \tilde{\alpha}\left(c_{2}+\gamma\right), \\
& S_{3}=C \alpha^{2} \\
& T_{1}=C \tilde{\beta} \\
& T_{2}=C^{2}+\beta \tilde{\beta}-\left(c_{2}+\gamma\right)^{2}, \\
& T_{3}=C \beta .
\end{aligned}
$$

If we now want to determine the critical electric field amplitude $\left|E_{c}\right|$ and the critical wave numbers $q_{x, c}$ and $q_{z, c}$, we must calculate the minimum of expression (21). The case of a LMW nematic liquid crystal is included in this expression; we obtain it by taking the limits $D_{1} \rightarrow 0$ and $D_{2} \rightarrow 0$ in (21) and in abbreviations (11)-(16), (22)-(27). This way we recover the classical bend Frederiks instability with a critical wave vector $\boldsymbol{q}_{\boldsymbol{c}}=\left(q_{x, c}, q_{z, c}\right)=\left(0, \pm \frac{\pi}{d}\right)$ and a critical electric field amplitude $\left|E_{c}\right|=\frac{\pi}{d} \sqrt{\frac{K_{3}}{\epsilon_{a}}}$ [11].

We can show that in the case of a nematic SCLSCE we can reduce the discussion of expression (21) to the one performed in reference [17] for the splay geometry. For this purpose, we note that by switching the material parameters $K_{1}$ and $K_{3}$, and by switching the sign of $D_{2}$, we obtain from expression (21) the corresponding expression for the splay geometry. In the present notation of this paper the expression for the splay geometry reads

$\epsilon_{a} E^{2}\left(q_{x}, q_{z}\right)=D_{1}+K_{3} q_{x}^{2}+K_{1} q_{z}^{2}-\frac{S_{3} q_{x}^{4}+S_{2} q_{x}^{2} q_{z}^{2}+S_{1} q_{z}^{4}}{T_{3} q_{x}^{4}+T_{2} q_{x}^{2} q_{z}^{2}+T_{1} q_{z}^{4}}$.

(This is expression (18) in Ref. [17], whereas we recall once again that the sign of $D_{2}$ has been defined in the opposite way in this reference.) As a consequence, the discussion in reference [17] for the splay geometry applies in the same way also for a nematic SCLSCE in the bend geometry for a material that has switched coefficients $K_{1}$ and $K_{3}$ as well as a switched sign of $D_{2}$.

From this analogy, we infer two important points. First, also in the bend geometry there can occur two qualitatively different instabilities at onset. One of them corresponds to the classical Frederiks instability and is characterized by $q_{x, c}=0$ and $q_{z, c}= \pm \frac{\pi}{d}$. It is illustrated on the right of Figure 2. The other one is undulatory with critical wave numbers $q_{x, c} \neq 0$ and $q_{z, c}= \pm \frac{\pi}{d}$. We depicted this qualitatively different instability on the right of Figure 3.

Second, we expect undulations to occur in the bend geometry at onset only for a positive sign of the material parameter $D_{2}$. This is contrary to the splay geometry, where we expect an undulatory instability only in the case of $D_{2}<0$. For an experiment this means the following: if undulations are detected at onset in the splay geometry,

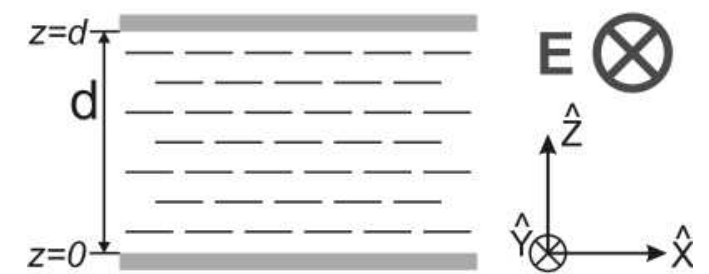

Fig. 4. Sketch of the ground-state director orientation of a nematic SCLSCE in the twist geometry.

but no undulations are found in the bend geometry, one can conclude that the sign of $D_{2}$ must be negative. If undulations are observed the other way around, the sign of $D_{2}$ has to be positive. Up to now, the value of the material parameter $D_{2}$ cannot be determined in an experiment directly.

We checked that surface energy contributions do not alter our results. The corresponding terms either vanish due to the boundary conditions, or the value of the wave number $q_{z}=q_{z, c}= \pm \frac{\pi}{d}$ leads to a cancellation of contributions resulting from different plate surfaces. In the case of the undulatory instability, the surface energy additionally vanishes separately on each plate.

\section{Twist geometry}

After reviewing the results derived for the splay geometry and studying the behavior of a nematic SCLSCE in the bend geometry, we now want to investigate the twist geometry. It is sketched in Figure 4. This geometry must be studied in all three dimensions. We take into account a homogeneous external electric field $\boldsymbol{E}=E \hat{\boldsymbol{y}}$, as well as equation (4) and

$$
\hat{\boldsymbol{n}}(z=0) \equiv \hat{\boldsymbol{n}}(z=d) \equiv\left(\begin{array}{l}
1 \\
0 \\
0
\end{array}\right) \equiv \hat{\boldsymbol{n}}_{0}
$$

as boundary conditions.

We then proceed in the same way as in the previous section. The independent variables corresponding to the macroscopic degrees of freedom are now all three components of the displacement field $u_{x}, u_{y}$, and $u_{z}$, as well as two components of the director field $n_{y}$ and $n_{z}$. From equation (3) we obtain the corresponding expression for the generalized energy density $F$ specified in Appendix B. From that we derive the five coupled partial differential equations that describe the actual state of the system, also listed explicitly in Appendix B: $\frac{\delta \mathcal{F}}{\delta u_{x}} \equiv 0, \frac{\delta \mathcal{F}}{\delta u_{y}} \equiv 0$, $\frac{\delta \mathcal{F}}{\delta u_{z}} \equiv 0, \frac{\delta \mathcal{F}}{\delta n_{y}} \equiv 0$, and $\frac{\delta \mathcal{F}}{\delta n_{z}} \equiv 0$.

As before, we use a Fourier ansatz in order to solve this system of equations:

$$
\begin{aligned}
& u_{x}=A \cos \left(q_{x} x+\vartheta_{x}\right) \cos \left(q_{y} y+\vartheta_{y}\right) \sin \left(q_{z} z\right), \\
& u_{y}=B \sin \left(q_{x} x+\vartheta_{x}\right) \sin \left(q_{y} y+\vartheta_{y}\right) \sin \left(q_{z} z\right), \\
& n_{y}=N \cos \left(q_{x} x+\vartheta_{x}\right) \sin \left(q_{y} y+\vartheta_{y}\right) \sin \left(q_{z} z\right), \\
& u_{z}=G \sin \left(q_{x} x+\vartheta_{x}\right) \cos \left(q_{y} y+\vartheta_{y}\right) \cos \left(q_{z} z\right), \\
& n_{z}=H \cos \left(q_{x} x+\vartheta_{x}\right) \cos \left(q_{y} y+\vartheta_{y}\right) \cos \left(q_{z} z\right) .
\end{aligned}
$$


Again, $A, B, N, G$, and $H$ are constant amplitudes in our linear stability analysis. We now have included the possibility of spatial undulations in all three dimensions, given by the wave numbers $q_{x}, q_{y}$, and $q_{z}$. By $\vartheta_{x}$ and $\vartheta_{y}$ we denote arbitrary phase angles (which, however, may not simply be set equal to zero without separately taking care of the cases $q_{x}=0$ and $q_{y}=0$, respectively).

The boundary conditions (4) and (29) demand $G=$ $H=0$, or equivalently

$$
\begin{aligned}
& u_{z} \equiv 0, \\
& n_{z} \equiv 0 .
\end{aligned}
$$

Introducing ansatz (30) into equations (B.2), (B.3), and (B.5), and by virtue of (31) and (32), we then obtain the following linear system of equations:

$$
\left(\begin{array}{ccc}
C q_{x}^{2}+\tilde{\beta}\left(q_{y}^{2}+q_{z}^{2}\right) & -\left(c_{2}+\gamma\right) q_{x} q_{y} & \tilde{\alpha} q_{y} \\
-\left(c_{2}+\gamma\right) q_{x} q_{y} & \beta q_{x}^{2}+C q_{y}^{2}+c_{1} q_{z}^{2} & -\alpha q_{x} \\
\tilde{\alpha} q_{y} & -\alpha q_{x} & M
\end{array}\right)\left(\begin{array}{c}
A \\
B \\
N
\end{array}\right)=0
$$

where

$$
M=D_{1}-\epsilon_{a} E^{2}+K_{1} q_{y}^{2}+K_{2} q_{z}^{2}+K_{3} q_{x}^{2},
$$

and where we have used the abbreviations (11)-(16). Besides the trivial solution $A=B=N=0$, corresponding to the ground state of the nematic SCLSCE, a nontrivial solution can be found if the determinant of the matrix vanishes. This leads us to the condition

$$
\epsilon_{a} E^{2}\left(q_{x}, q_{y}, q_{z}\right)=K_{1} q_{y}^{2}+K_{2} q_{z}^{2}+K_{3} q_{x}^{2}+D_{1}-\frac{\Xi_{1}}{\Xi_{2}},
$$

with the abbreviations

$$
\begin{aligned}
\Xi_{1}= & \left(C \alpha^{2}\right) q_{x}^{4}+\left(C \tilde{\alpha}^{2}\right) q_{y}^{4} \\
& +\left[\alpha^{2} \tilde{\beta}-2\left(c_{2}+\gamma\right) \alpha \tilde{\alpha}+\tilde{\alpha}^{2} \beta\right] q_{x}^{2} q_{y}^{2} \\
& +\left(\tilde{\beta} \alpha^{2}\right) q_{x}^{2} q_{z}^{2}+\left(c_{1} \tilde{\alpha}^{2}\right) q_{y}^{2} q_{z}^{2}, \\
\Xi_{2}= & (C \beta) q_{x}^{4}+(C \tilde{\beta}) q_{y}^{4}+\left(c_{1} \tilde{\beta}\right) q_{z}^{4} \\
& +\left[C^{2}+\beta \tilde{\beta}-\left(c_{2}+\gamma\right)^{2}\right] q_{x}^{2} q_{y}^{2} \\
& +\left(C c_{1}+\beta \tilde{\beta}\right) q_{x}^{2} q_{z}^{2}+\left(\tilde{\beta} c_{1}+\tilde{\beta} C\right) q_{y}^{2} q_{z}^{2} .
\end{aligned}
$$

Before we continue with the determination of the critical wave numbers and thus the minimum of expression (35), we have a short look at the remaining Frank energy density in equation (B.1),

$$
F_{\text {Frank }}=\frac{1}{2} K_{1}\left(\partial_{y} n_{y}\right)^{2}+\frac{1}{2} K_{2}\left(\partial_{z} n_{y}\right)^{2}+\frac{1}{2} K_{3}\left(\partial_{x} n_{y}\right)^{2} .
$$

We can see that an $x$-, $y$-, $z$-dependence of $n_{y}$ corresponds to a bend, splay, twist deformation of the director field, respectively. Consequently, three-dimensional solutions include splay, bend, as well as twist deformation of the director field, and they will be referred to as class (I) in the following. We will also obtain two-dimensional solutions characterized by $q_{x}=0$, which only contain splay and twist deformation and will be denoted as class (II); and two-dimensional solutions of $q_{y}=0$ that describe a bend and twist deformation will be referred to as class (III).

The explicit determination of candidates for the critical wave numbers is presented in Appendix C. It turns out that the critical wave number $q_{z, c}$ is given by $q_{z, c}= \pm \frac{\pi}{d}$. Consequently, we have to find the global minimum of the surface $\epsilon_{a} E^{2}\left(q_{x}, q_{y}, \pm \frac{\pi}{d}\right)$ over the $\left(q_{x}, q_{y}\right)$-plane. There are three candidates for this minimum: one of them is given by $\boldsymbol{q}^{(\mathrm{I})}=\left(0,0, \pm \frac{\pi}{d}\right)$ in the framework of our linear stability analysis. In general we must assume that the corresponding minimum is located at $\left(q_{x, c}, q_{y, c}, \pm \frac{\pi}{d}\right)$, where $q_{x, c}^{2}, q_{y, c}^{2} \ll q_{z, c}^{2}$, including undulations in both lateral directions and thus belonging to class (I). The other two candidates will be called $\boldsymbol{q}^{\text {(II) }}$ with $q_{x, c}=0$ as given by equation (C.7), and $\boldsymbol{q}^{(\mathrm{III})}$ with $q_{y, c}=0$ as noted in equation (C.9). In addition, we know from equation (35) that

$$
\epsilon_{a} E^{2}\left(q_{x}, q_{y}, \pm \frac{\pi}{d}\right) \rightarrow \infty \quad \text { for } q_{x} \rightarrow \infty \quad \text { or } \quad q_{y} \rightarrow \infty
$$

which restricts our discussion to these three candidates for the minimum. From the analysis of the Hessian (C.3) in Appendix $\mathrm{C}$ we know that the sample thickness $d$ is the crucial parameter which determines the location of the minimum in the $\left(q_{x}, q_{y}\right)$-plane. There exist two assigned values $d_{1}$ and $d_{2}$ given by equations (C.4) and (C.5), which separate the qualitative appearance of the instabilities.

To get a final overview, we investigate what happens for an increasing sample thickness $d$, and we illustrate our results by an example. For the latter purpose we choose numerical values for the material parameters involved such that they satisfy the thermodynamic stability conditions: $c_{1}=10^{4} \mathrm{~J} \mathrm{~m}^{-3}, c_{2}=5 \times 10^{8} \mathrm{~J} \mathrm{~m}^{-3}, D_{1}=10^{3} \mathrm{~J} \mathrm{~m}^{-3}, D_{2}=$ $5 \times 10^{2} \mathrm{~J} \mathrm{~m}^{-3}, K_{1}=10^{-11} \mathrm{~J} \mathrm{~m}^{-1}, K_{2}=7 \times 10^{-12} \mathrm{~J} \mathrm{~m}^{-1}$, and $K_{3}=5 \times 10^{-11} \mathrm{~J} \mathrm{~m}^{-1}$.

i) Small sample thickness $d<d_{1}$ and simultaneously $d<d_{2}$ :

$\boldsymbol{q}^{(\mathrm{I})}$ is the only existing candidate for a minimum. The Hessian (C.3) confirms that at $\boldsymbol{q}^{(\mathrm{I})}$ a minimum occurs, and due to the limit (39) this must be the global minimum of $\epsilon_{a} E^{2}\left(q_{x}, q_{y}, \pm \frac{\pi}{d}\right)$. As discussed before, the observed instability at onset may in general be three dimensional with undulations in both lateral directions.

Calculating the corresponding critical electric field amplitude, we obtain the expression

$$
\epsilon_{a} E_{c}^{2}=D_{1}+K_{2}\left(\frac{\pi}{d}\right)^{2} .
$$

This is a very important result because it offers the possibility of determining the values of the material parameters $D_{1}$ and $K_{2}$ in an experiment. For this purpose, samples of one nematic SCLSCE of different, but small, thicknesses $d$ would have to be probed in the twist geometry. As $\epsilon_{a}$ is known or can be estimated, from a plot of $\epsilon_{a} E_{c}^{2}$ versus the inverse of $d^{2}$ the values of $D_{1}$ and $K_{2}$ could be inferred. Up to now, no experimental set-up is known that allows to measure $D_{1}$ directly. Also the value of the Frank coefficient $K_{2}$ to our knowledge has not been determined for a nematic SCLSCE in an experiment yet. 


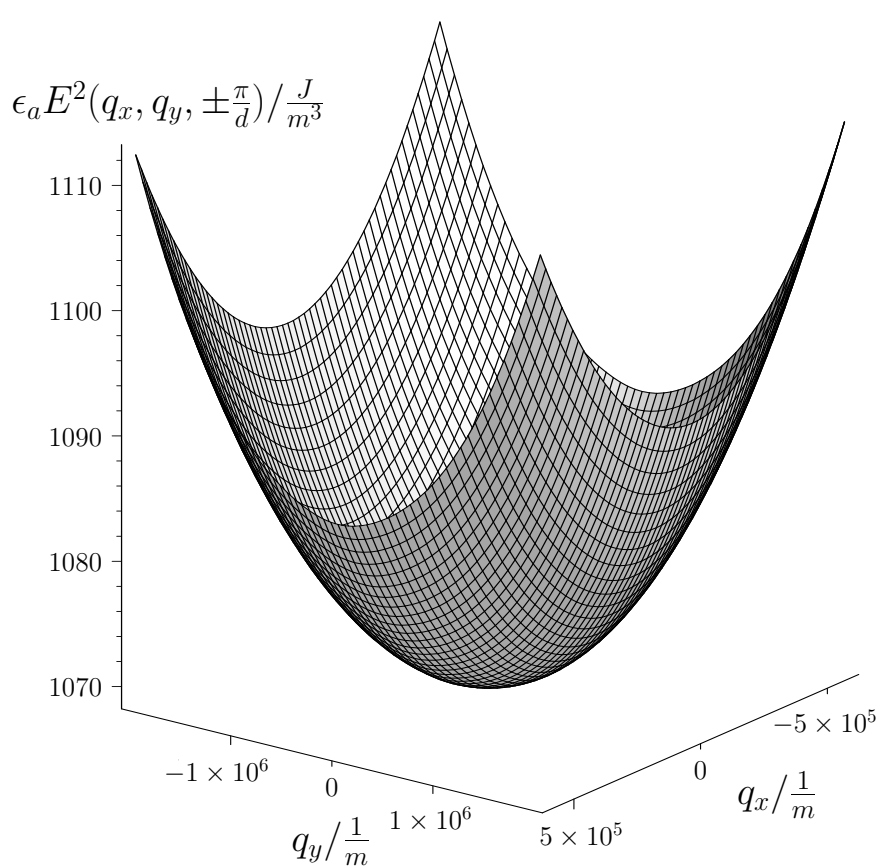

Fig. 5. $\epsilon_{a} E^{2}\left(q_{x}, q_{y}, \pm \frac{\pi}{d}\right)$ over the $\left(q_{x}, q_{y}\right)$-plane, where $d=$ $1 \mu \mathrm{m}$ and the material parameters chosen as noted in the text.

Turning to our example with the numerical values of the material parameters selected as noted above, we obtain the two limiting values for the sample thickness: $d_{1} \approx 1.4 \mu \mathrm{m}$ and $d_{2} \approx 8.9 \mu \mathrm{m}$. If we choose, for example, a sample thickness of $d=1 \mu \mathrm{m}$, the Maple plot in Figure 5 confirms that there exists a global minimum at $\boldsymbol{q}^{(\mathrm{I})}=\left(0,0, \pm \frac{\pi}{d}\right)$.

ii) Intermediate sample thickness $d_{1} \leq d<d_{2}$ :

We now obtain as a second candidate for a minimum the point $\boldsymbol{q}^{(\mathrm{II})}$. For $d=d_{1}$, the two points $\boldsymbol{q}^{(\mathrm{I})}$ and $\boldsymbol{q}^{(\mathrm{II})}$ are degenerate, but for increasing sample thickness they separate from each other. Simultaneously, the Hessian (C.3) shows us that $\boldsymbol{q}^{(\mathrm{I})}$ now has become a saddle point which is metastable with respect to the $q_{y}$-direction. On the contrary, from the Hessian it follows that a minimum can be found at $\boldsymbol{q}^{(\mathrm{II})}$. Therefore the global minimum of $\epsilon_{a} E^{2}\left(q_{x}, q_{y}, \pm \frac{\pi}{d}\right)$ is not found at $\boldsymbol{q}^{(\mathrm{I})}$ any longer but at $\boldsymbol{q}^{(\mathrm{II})}$. We thus observe a two-dimensional undulatory instability of splay and twist deformation at onset at a critical electric field amplitude given by

$$
\epsilon_{a} E_{c}^{2}<D_{1}+K_{2}\left(\frac{\pi}{d}\right)^{2}
$$

Of course this case can only occur if $d_{1}<d_{2}$.

For illustration we now choose the value $d=5 \mu \mathrm{m}$ such that $d_{1}<d<d_{2}$, and we find the surface depicted in Figure 6. As expected and described by the Hessian (C.3), $\boldsymbol{q}^{(\mathrm{I})}$ now has turned into a saddle point. However, the surface has only become metastable at $\boldsymbol{q}^{(\mathrm{I})}$ in $q_{y}$-direction, in $q_{x}$-direction it remains stable. Furthermore, we now clearly identify the new minima at $\boldsymbol{q}^{(\mathrm{II})}$, where $q_{x}=0$ but $q_{y} \neq 0$.

iii) Intermediate sample thickness $d_{1}>d \geq d_{2}$ : It goes without saying that this situation can only occur

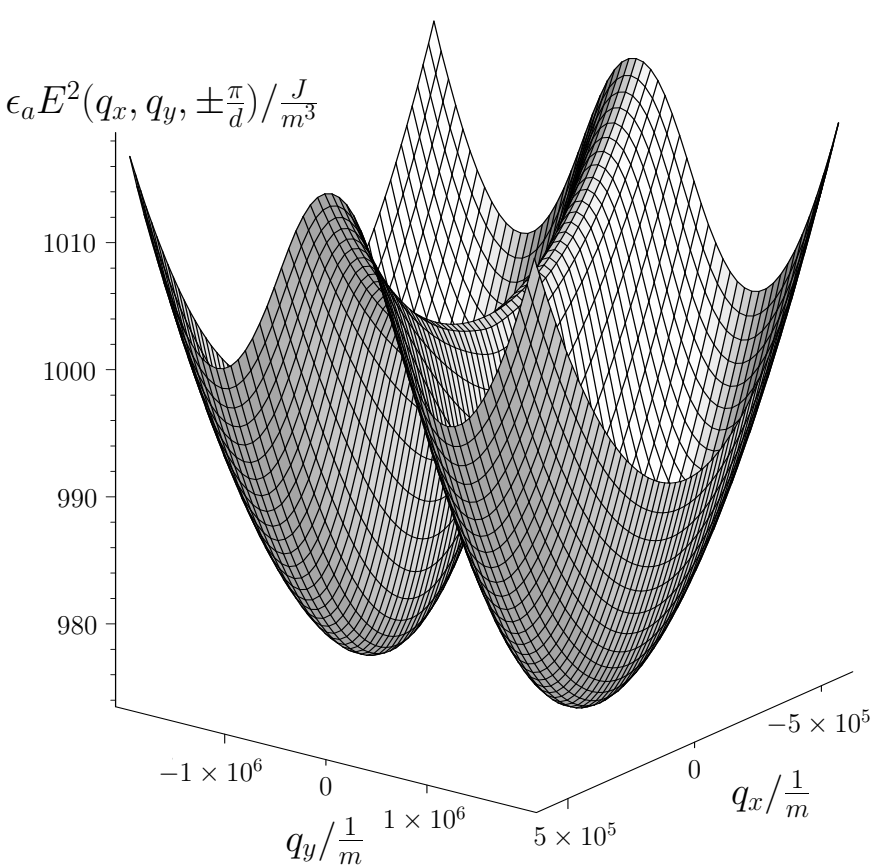

Fig. 6. $\epsilon_{a} E^{2}\left(q_{x}, q_{y}, \pm \frac{\pi}{d}\right)$ over the $\left(q_{x}, q_{y}\right)$-plane with $d=5 \mu \mathrm{m}$.

if $d_{1}>d_{2}$. Consequently, it cannot be realized in the same material that could be used in order to study the previous case. However, the situation is formally analogous to the one treated before in ii). The new global minimum is now described by $\boldsymbol{q}^{(\mathrm{III})}$ and it emerges at $d=d_{2}$. For increasing $d$ it separates from $\boldsymbol{q}^{(\mathrm{I})}$ in $q_{x}$-direction. It characterizes a two-dimensional undulatory instability that involves a bend and twist deformation of the director field at onset.

iv) Large sample thickness $d \geq d_{1}$ and simultaneously $d \geq d_{2}$ :

For these large sample thicknesses the Hessian (C.3) shows us that the surface becomes metastable at $\boldsymbol{q}^{(\mathrm{I})}$ in $q_{x^{-}}$as well as in $q_{y}$-direction. So we now find a competition between the two minima at $\boldsymbol{q}^{(\mathrm{II})}$ and $\boldsymbol{q}^{(\mathrm{III})}$, which leads either to a splay and twist or a bend and twist deformation at onset. In both cases we observe a two-dimensional undulatory instability.

In order to determine the position of the global minimum we have to find out whether either $\epsilon_{a} E^{2}\left(\boldsymbol{q}^{(\mathrm{II})}\right)$ or $\epsilon_{a} E^{2}\left(\boldsymbol{q}^{(\mathrm{III})}\right)$ has the lower value. For this purpose we insert expressions (C.7) and (C.9) into equations (35)-(37), and we investigate $\epsilon_{a} E^{2}\left(\boldsymbol{q}^{(\mathrm{II})}\right)-\epsilon_{a} E^{2}\left(\boldsymbol{q}^{(\mathrm{III})}\right)$. The latter expression can be simplified with the help of the binomial theorem and by taking a square root, remembering that $d \geq d_{1}$ and $d \geq d_{2}$. We find that in the case of

$$
\frac{\pi}{d}\left(\sqrt{K_{1}}-\sqrt{\frac{K_{3} c_{1}}{\beta}}\right)+\left(\frac{|\alpha|}{\sqrt{\beta}}-\frac{|\tilde{\alpha}|}{\sqrt{\tilde{\beta}}}\right)<0
$$

the global minimum is located at $\boldsymbol{q}^{(\mathrm{II})}$ so that we observe an undulatory splay and twist deformation at onset. Otherwise we find the global minimum at $\boldsymbol{q}^{(\mathrm{III})}$, which coincides with an undulatory bend and twist deformation. 


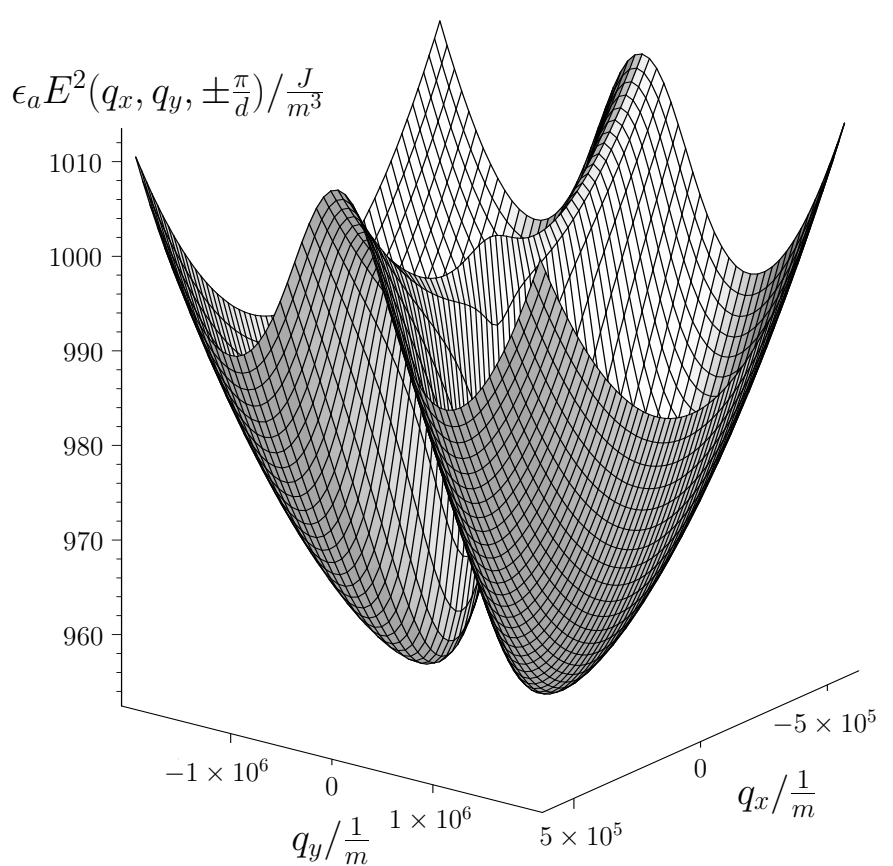

Fig. 7. $\epsilon_{a} E^{2}\left(q_{x}, q_{y}, \pm \frac{\pi}{d}\right)$ over the $\left(q_{x}, q_{y}\right)$-plane with $d=$ $20 \mu \mathrm{m}$.

For large sample thicknesses the second term in equation (42) dominates. We insert the abbreviations (12)-(15) and use the fact that $\beta>0$ and $\tilde{\beta}>0$, which results from the thermodynamic stability conditions listed at the end of Section 2. This way, inequality (42) simplifies to $\left(4 c_{1} D_{1}-D_{2}^{2}\right) D_{2}>0$, and further, taking advantage of the thermodynamic stability condition $D_{2}^{2}<4 c_{1} D_{1}$, to

$$
D_{2}>0
$$

Thus, for large sample thicknesses, only the sign of $D_{2}$ determines the kind of instability we observe at onset. For $D_{2}>0$ we expect splay and twist undulations at onset in $y$-direction with $q_{x}=0$, and for $D_{2}<0$ bend and twist undulations in $x$-direction with $q_{y}=0$. Again, this is an important result, because this way one should be able to infer the sign of $D_{2}$ from the experimental observation of a thick sample of a nematic SCLSCE in the twist geometry. We must add that here "large" sample thickness means that $d \gg d_{1}$ for $D_{2}>0$, and $d \gg d_{2}$ in the case of $D_{2}<0$. When in addition we require $d \gg \pi \sqrt{K_{2} / D_{1}}$ as well as either $d \gg \pi^{2} K_{1} /\left(D_{1} d_{1}\right)$ in the case of $D_{2}>0$ or $d \gg \pi^{2} K_{3} c_{1} /\left(D_{1} \beta d_{2}\right)$ for $D_{2}<0$, the critical external electric field amplitude is given by

$$
\epsilon_{a} E_{c}^{2}=\frac{4 c_{1} D_{1}-D_{2}^{2}}{4 c_{1}+D_{1}+2\left|D_{2}\right|} .
$$

This is also a very interesting result concerning the so far unknown values of the material parameters. As we have shown, from measurements of $\epsilon_{a} E^{2}$ for small sample thicknesses, the value of the material parameter $D_{1}$ could be derived. $c_{1}$ can be determined from classical elasticity measurements. Measuring the critical electric field amplitude for large sample thicknesses $d$ should then offer the

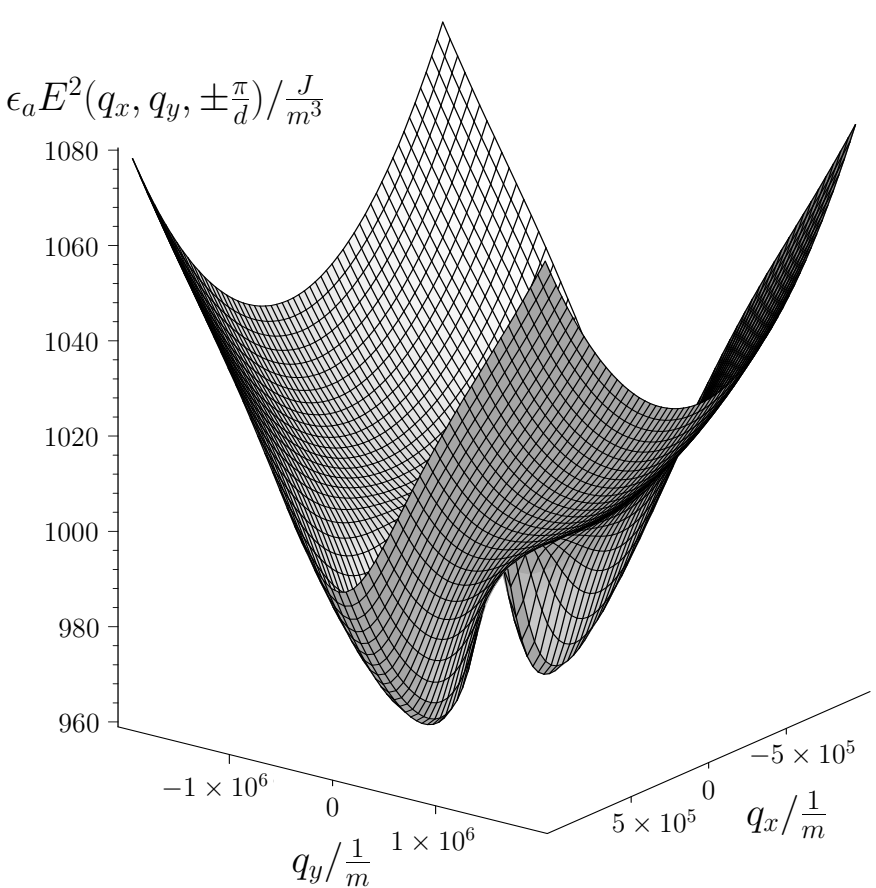

Fig. 8. $\epsilon_{a} E^{2}\left(q_{x}, q_{y}, \pm \frac{\pi}{d}\right)$ over the $\left(q_{x}, q_{y}\right)$-plane with $d=$ $20 \mu \mathrm{m}, D_{2}=-5 \times 10^{2} \mathrm{~J} \mathrm{~m}^{-3}$, and the values of the other material parameters as noted in the text.

possibility to estimate the absolute value of $D_{2}$ from equation (44). According to equation (44), $\epsilon_{a} E_{c}^{2}$ should become independent of the exact value of $d$ for large sample thicknesses. This means that the influence of the boundaries becomes negligible and the coupling of the director to the polymer matrix dominates. The sign of $D_{2}$ follows from the quality of the two-dimensional instability observed, which we have explained above.

Finally, turning to our example, we choose a sample thickness of $d=20 \mu \mathrm{m}$. For this value we obtain the surface depicted in Figure 7, in which we find the global minimum located on the line $q_{x}=0$ as before in Figure 6. In contrast to Figure 6, the surface now has become metastable at the point $\boldsymbol{q}^{(\mathrm{I})}$ in both directions of $q_{x}$ and $q_{y}$, reflecting the behavior of the Hessian (C.3).

When we have a look at the two terms of inequality (42), we notice that for the numerical values of our example the second term is already about one order of magnitude larger than the first one $\left(-4.8 \sqrt{\mathrm{J} / \mathrm{m}^{3}}\right.$ versus $\left.-0.6 \sqrt{J / m^{3}}\right)$. In this case, as noted above, the sign of $D_{2}$ should lead to a splay and twist deformation with $q_{x, c}=0$. Figure 7 confirms this relation. In order to verify the importance of the sign of $D_{2}$ for the quality of the twodimensional undulatory instability, we switch the sign of $D_{2}$ such that now $D_{2}=-5 \times 10^{2} \mathrm{~J} \mathrm{~m}^{-3}$ and all the other numerical values remain the same. After that, we expect an undulatory instability of bend and twist with $q_{y, c}=0$ to occur at onset, which is confirmed by the plot of the surface $\epsilon_{a} E^{2}\left(q_{x}, q_{y}, \pm \frac{\pi}{d}\right)$ in Figure 8 .

We have added Appendix D, in which we shortly discuss what happens if the first term dominates in inequality (42). In addition, we would like to mention that our 
results are not altered by the influence of any contributions to a generalized surface energy. Due to ansatz (30), together with equations (31), (32), and (C.2), no such contributions arise at the surface of our system.

At the end of this section, we want to check the consistency of our model with the low molecular weight case. Therefore, we take the limit $D_{1} \rightarrow 0$ and $D_{2} \rightarrow 0$, which corresponds to a decoupling of the liquid crystalline subsystem from the elastic one. Equation (35) then reduces to

$$
\epsilon_{a} E^{2}\left(q_{x}, q_{y}, q_{z}\right)=K_{1} q_{y}^{2}+K_{2} q_{z}^{2}+K_{3} q_{x}^{2}
$$

so that we obtain $\boldsymbol{q}_{\boldsymbol{c}}=\left(0,0, \pm \frac{\pi}{d}\right)$ and

$$
E_{c}=\frac{\pi}{d} \sqrt{\frac{K_{2}}{\epsilon_{a}}} .
$$

In addition to the identities (31) and (32), we now find that the components $u_{x}$ and $u_{y}$ vanish because they decouple in the system of equations (B.2)-(B.6). Consequently, the instability is characterized by

$$
u_{x} \equiv u_{y} \equiv u_{z} \equiv n_{z} \equiv 0 \quad \text { and } \quad n_{y}=N \sin \left(\frac{\pi}{d}\right)
$$

and we have thus recovered the LMW Frederiks transition in the twist geometry [11].

\section{Summary, discussion and conclusion}

In this paper, the behavior of nematic SCLSCEs exposed to an external electric or magnetic field in the bend and in the twist geometry has been studied. The results obtained in reference [17] for the splay geometry have been reviewed briefly. The investigations have been performed by means of a linearized continuum model, and as a result, laterally homogeneous Frederiks-like instabilities as well as qualitatively different laterally undulated instabilities have been found.

Whereas undulations in liquid crystalline polymers exposed to an external electric or magnetic field have already been observed and studied in the splay geometry (see, e.g., $[26,27])$, the undulatory instabilities found and investigated in reference [17] and in this paper are qualitatively different and to our knowledge have not been noted elsewhere for nematic SCLSCEs before. They are static and directly result from the coupling of the liquid crystalline subsystem to the elastic one, or, in other words, from the coupling of the macroscopic mesogenic orientational behavior to the macroscopic elastic behavior of the polymer network. In reference [18] we have given an explanation for the fact that this coupling can lead to undulations.

A splay geometry has also been investigated by other authors in reference [15], using a different model. When we compare their results for the splay geometry to our results, we find qualitative differences. The analysis in reference [15] suggests a variation of the director field only in $z$-direction, where a variation of full wavelength occurs between the plate surfaces. At onset, this would correspond to a critical wave number $q_{z, c}=\frac{2 \pi}{d}$ in the Fourier analysis. Lateral undulations have not been found in reference [15].

We note that major differences exist between the analysis performed in reference [15] on the one hand and ours on the other hand. Whereas the elastomer was completely fixed at the boundaries in reference [15], it was allowed to slip along the plate surfaces in our case. As a consequence we find a critical wavenumber $q_{z, c}=\frac{\pi}{d}$ in Section 3. When we fix the elastomer at the boundaries, that is we set $u_{z} \equiv 0$ at the plate surfaces, we find $q_{z, c}=\frac{2 \pi}{d}$ as a critical mode in our Fourier analysis for the splay and bend geometry. Therefore, the boundary conditions play a crucial role for the behavior of the materials. In contrast, in the twist geometry the solutions represented by ansatz (30) as well as equations (31) and (32) show a vanishing displacement field $\boldsymbol{u} \equiv \mathbf{0}$ on the plate surfaces in any case. Lateral undulations could not be found in reference [15], and also not in the numerical investigation [16], because there lateral spatial variations were excluded.

Furthermore, the analysis in reference [15] is based on a different, mesoscopic approach. The elastic part of the energy density is derived from a model of rubber elasticity. In this connection, the liquid crystalline properties are taken into account by the assumption of an anisotropic Gaussian distribution for the arrangement of the polymer segments. This model contains nonlinearities and therefore the analysis is also extended to electric field amplitudes well above threshold. On the contrary, the model we use in order to investigate the macroscopic behavior of the materials is based on a formulation in the hydrodynamic spirit. Macroscopic variables reflecting the influence of the two components of the system and a coupling between both are combined by symmetry arguments. As a result, we obtain an expression for the energy density which clearly distinguishes between one subsystem showing elastic behavior, one subsystem reflecting the properties of the liquid crystalline state, and the coupling between both components. The present notation of the model is a linear one, so it is restricted to describe the behavior of the materials in the immediate vicinity of the threshold.

It has already been noted in the two preceding sections that the conclusions which could be drawn from the experimental studies of nematic SCLSCEs in the three geometries would be of major importance. First of all, it would be very interesting to observe all the instabilities which up to now have only been predicted theoretically in this paper. Second, the values of the twist coefficient $K_{2}$ and of the material parameters $D_{1}$ and $D_{2}$ connected to the variables of relative rotations could be inferred from the experimental results. As we have explained, the sign of $D_{2}$ can be extracted from the observation of undulations at onset either in the splay or in the bend geometry as well as from the direction of the two-dimensional undulations occurring in twist experiments of large sample thickness. Furthermore, $K_{2}, D_{1}$, and the absolute value of $D_{2}$ could be obtained from measurements of the critical external field amplitude for samples of different thicknesses in the twist geometry. Thereby, experiments of small sample thicknesses can give the values of $K_{2}$ and $D_{1}$, and from 
experiments of large sample thicknesses $\left|D_{2}\right|$ could be evaluated in a second step. This is very important because up to now no experimental set-up is known to determine the values of $D_{1}$ and $D_{2}$. The same applies for the determination of the value of the coefficient $K_{2}$, which is not connected to any explicit coupling between the director field and the polymer network.

We would like to stress at this point that to us it seems as if a twist geometry was the only suitable geometry in order to measure the material parameter $D_{1}$ directly. $D_{1}$ is connected to that contribution of the generalized energy density which only results from a relative rotation between the two subsystems. If the entity of the mesogenic units is rotated with respect to the polymer matrix, a relative rotation results and thus an energetic contribution arises from the $D_{1}$-term. In order to probe this contribution in an experiment, one has to fix, for example, the director orientation and rotate the polymer network against the average orientation of the mesogenic units. The director can be fixed in the bulk only by an electric or magnetic field, whereas the rotation of the polymer network can only be enforced by applying mechanical torques at the sample boundaries. Of course we obtain an equivalent situation when we try to keep the polymer network fixed by the boundary conditions and reorient the director by applying an external electric or magnetic field. However, in the linear regime of reorienting the director field, this corresponds to the set-up for the twist geometry investigated in this paper. Measuring the critical external field amplitude for a reorientation of the director field shown by an instability which is nearly homogeneous in lateral directions, it should then be possible to determine $D_{1}$ in an experiment directly. We have demonstrated in Section 4 that for small sample thicknesses such an instability arises in the twist geometry. In addition we have shown that by plotting the critical external field amplitude versus the inverse squared sample thickness, the influence of the strong anchoring boundary conditions for the director field can be separated and the value of $D_{1}$ can be inferred.

The experimental study of the behavior of nematic SCLSCEs in the three geometries and the observation of the Frederiks-like and undulatory instabilities at onset is still an open field, especially concerning the bend and twist geometry. In reference [12] the authors studied a swollen nematic SCLSCE in the splay geometry, and they found an "irregular wiggled profile" in the direction of the ground-state director orientation. Possibly, at onset this corresponds to our undulatory instability and thus might be its first experimental observation.

It is difficult to further specify the circumstances under which the undulatory instabilities can be observed due to the undetermined numerical values of several of the material parameters. Nevertheless, on the basis of our previous studies [18], we can say that a lower value of the elastic modulus $c_{1}$ adds to the formation of the lateral undulations. From further analysis we can conclude that undulations in the splay geometry should be searched for in materials that show a reorientation of the director field when being stretched perpendicularly to the initial director orientation $\left(D_{2}<0\right)$. Undulations in the bend geometry should be searched for in materials that do not exhibit director reorientation under these circumstances $\left(D_{2}>0\right)$.

During our discussion we must not forget that common nematic SCLSCEs are quite heterogeneous materials. Recently, this fact has been confirmed by NMR and calorimetry measurements $[7,28]$, and we therefore have to be aware that nematic SCLSCEs show spatial variations of their initial director orientation. On the contrary, in our calculations we have assumed an ideal spatially homogeneous initial orientation of the director field. This has led us to the laterally homogeneous or undulated solutions, imposing strong anchoring of the director orientation at the sample surfaces. Strong anchoring boundary conditions stabilize a spatially homogeneous initial director orientation and therefore favor the occurrence of our solutions in an experimental realization of the geometries. Furthermore, our previous investigations show that the wavelengths of the lateral undulations are of the order of the sample thickness [18]. Especially for thicker samples this macroscopic length becomes considerably larger than the typical length scale expected for the inherent spatial variation of the material properties. Therefore it should be possible to observe the lateral undulations in an experiment in spite of the spatial inhomogeneities of the materials.

We note that the amplitudes of the external electric or magnetic field have to be comparatively large in order to observe the reorientation effects in common nematic SCLSCEs. However, the corresponding values should be achievable in experiments nowadays. Furthermore, swelling nematic SCLSCEs with common LMWLCs as was done for example in references $[10,12]$ drastically reduces the necessary field amplitudes. Our results should describe the effects observed in these materials in the same way, as long as electric currents do not lead to dominating different effects and as long as the different mesogens of the SCLSCE and the LMWLC show the same behavior of reorientation.

In our considerations we have always assumed the external field to be static. However, our results should also be valid for external fields that vary slowly in time. Here, "slow" means that the orientation of the director field and the distortion of the elastomer following from the coupling of the director to the polymer network must always adopt a state close to equilibrium for the present external field amplitude. On the other hand, our results should also be essential for the experimental investigation of nematic side-chain liquid crystalline polymers in periodically varying external electric or magnetic fields. In this case, one must make sure that the polymer chains are long enough so that the mechanical response of the material is dominated by the elastic behavior of the transient polymer network.

Another issue we would like to address is the experimental finding that nematic SCLSCEs are to a very good approximation incompressible materials. In our model this means that the material parameter $c_{2}$ tends to infinity. We 
kept our description general and did not explicitly take this limit for the value of $c_{2}$ in our calculations. However, this can easily be done and does not change our results qualitatively. In the expressions for the external field amplitudes (21) and (35), $c_{2}$ enters the numerator and denominator of the fractions at equal order and thus cancels without leading to any divergence. The values of the sample thickness that determine the quality of the instabilities in the twist geometry do not depend on $c_{2}$, as can be seen from inequalities (C.4) and (C.5).

On the whole, it would be of major importance to perform the experiments proposed in this paper. This would increase our understanding of the materials and certainly be useful from the point of view of applications. Furthermore, expanding the numerical studies as the one carried out in reference [16] by including the possibility of undulations in lateral directions would be very interesting. Finally, extending the linearized description of the geometries investigated in this paper to the nonlinear regime is another issue for future work. Recently, nonlinear expressions for the variables of relative rotations have been derived [25], and their inclusion into nonlinear studies may reveal new, qualitatively different effects.

We thank the Deutsche Forschungsgemeinschaft through the Forschergruppe FOR608 "Nichtlineare Dynamik komplexer Kontinua" and the Deutscher Akademischer Austauschdienst (312/pro-ms) through PROCOPE for partial support of this work.

\section{Appendix A. Vanishing influence of the flexoelectric terms}

In this Appendix, we demonstrate that the flexoelectric terms listed in equation (3) with the coefficients $e_{1}$ and $e_{3}$ do not contribute to the solutions found.

Studying the bend geometry $\hat{\boldsymbol{n}}_{0} \| \hat{\boldsymbol{z}}$ and $\boldsymbol{E}=E \hat{\boldsymbol{x}}$, the flexoelectric terms in equation (3) up to quadratic order turn into

$$
-e_{1} E\left(n_{x} \partial_{x}\right) n_{x}-e_{3} E\left(n_{x} \partial_{x}+\partial_{z}\right) n_{x}
$$

We can see that they vanish in the bulk equation (10) after performing the variational derivative with respect to $n_{x}$. On the contrary, from equation (19), we obtain a contribution to the surface energy density that reads

$$
-e_{3} E\left(\partial_{z} n_{x}\right)=-e_{3} E q_{z} N \sin \left(q_{x} x+\vartheta\right) \cos \left(q_{z} z\right) .
$$

However, in the case of the instabilities studied, the wave number $q_{z}$ always turned out to be $q_{z, c}= \pm \frac{\pi}{d}$. This means that the flexoelectric contributions at different plate surfaces cancel each other. In addition, for an undulatory instability, the flexoelectric contribution to the surface energy vanishes on each surface separately.

In the twist geometry $\hat{\boldsymbol{n}}_{0} \| \hat{\boldsymbol{x}}$ and $\boldsymbol{E}=E \hat{\boldsymbol{y}}$, we obtain from equation (3) up to quadratic order the terms

$$
-e_{1} E n_{y}\left(\partial_{y} n_{y}+\partial_{z} n_{z}\right)-e_{3} E\left(\partial_{x}+n_{y} \partial_{y}+n_{z} \partial_{z}\right) n_{y} .
$$

This leads to a contribution

$$
-\left(e_{1}-e_{3}\right) E\left(\partial_{z} n_{z}\right)
$$

in the bulk equation resulting from the variational derivative of $\mathcal{F}$ with respect to $n_{y}$, and to a contribution

$$
\left(e_{1}-e_{3}\right) E\left(\partial_{z} n_{y}\right)
$$

in the corresponding variational derivative of $\mathcal{F}$ with respect to $n_{z}$. However, due to the boundary conditions, we found $n_{z} \equiv 0$ so that expression (A.4) is equal to zero. On the contrary, expression (A.5) does not vanish. But since $n_{z} \equiv 0$, the variation of $\mathcal{F}$ with respect to $n_{z}$ loses its meaning. Furthermore, due to the boundary conditions, there are no flexoelectric contributions to the generalized surface energy density.

For this reason, the description of the geometries investigated is indeed formally identical for an external electric and an external magnetic field.

\section{Appendix B. Equations describing the twist geometry}

Here, we list the explicit expression for the generalized energy density $F$ characterizing a nematic SCLSCE in the twist geometry:

$$
\begin{aligned}
& F=F_{0}+\frac{1}{2} c_{1}\left\{2\left(\partial_{x} u_{x}\right)^{2}+2\left(\partial_{y} u_{y}\right)^{2}+2\left(\partial_{z} u_{z}\right)^{2}\right. \\
& +\left[\left(\partial_{x} u_{y}\right)+\left(\partial_{y} u_{x}\right)\right]^{2}+\left[\left(\partial_{x} u_{z}\right)+\left(\partial_{z} u_{x}\right)\right]^{2} \\
& \left.+\left[\left(\partial_{y} u_{z}\right)+\left(\partial_{z} u_{y}\right)\right]^{2}\right\} \\
& +\frac{1}{2} c_{2}\left\{\left(\partial_{x} u_{x}\right)+\left(\partial_{y} u_{y}\right)+\left(\partial_{z} u_{z}\right)\right\}^{2} \\
& +\frac{1}{2} D_{1}\left\{n_{y}^{2}-n_{y}\left[\left(\partial_{x} u_{y}\right)-\left(\partial_{y} u_{x}\right)\right]\right. \\
& +\frac{1}{4}\left[\left(\partial_{x} u_{y}\right)-\left(\partial_{y} u_{x}\right)\right]^{2} \\
& +n_{z}^{2}-n_{z}\left[\left(\partial_{x} u_{z}\right)-\left(\partial_{z} u_{x}\right)\right] \\
& \left.+\frac{1}{4}\left[\left(\partial_{x} u_{z}\right)-\left(\partial_{z} u_{x}\right)\right]^{2}\right\} \\
& +\frac{1}{2} D_{2}\left\{n_{y}\left[\left(\partial_{x} u_{y}\right)+\left(\partial_{y} u_{x}\right)\right]-\frac{1}{2}\left(\partial_{x} u_{y}\right)^{2}\right. \\
& +\frac{1}{2}\left(\partial_{y} u_{x}\right)^{2}+n_{z}\left[\left(\partial_{x} u_{z}\right)+\left(\partial_{z} u_{x}\right)\right] \\
& \left.-\frac{1}{2}\left(\partial_{x} u_{z}\right)^{2}+\frac{1}{2}\left(\partial_{z} u_{x}\right)^{2}\right\} \\
& -\frac{1}{2} \epsilon_{a} E^{2} n_{y}^{2} \\
& +\frac{1}{2} K_{1}\left\{\left(\partial_{y} n_{y}\right)^{2}+2\left(\partial_{y} n_{y}\right)\left(\partial_{z} n_{z}\right)+\left(\partial_{z} n_{z}\right)^{2}\right\} \\
& +\frac{1}{2} K_{2}\left\{\left(\partial_{y} n_{z}\right)^{2}-2\left(\partial_{y} n_{z}\right)\left(\partial_{z} n_{y}\right)+\left(\partial_{z} n_{y}\right)^{2}\right\} \\
& +\frac{1}{2} K_{3}\left\{\left(\partial_{x} n_{y}\right)^{2}+\left(\partial_{x} n_{z}\right)^{2}\right\} \text {. }
\end{aligned}
$$


Minimizing the corresponding generalized energy $\mathcal{F}$ leads to the following five coupled differential equations:

$$
\begin{gathered}
0 \equiv \frac{\delta \mathcal{F}}{\delta u_{x}} \equiv-c_{1}\left\{2\left(\partial_{x}^{2} u_{x}\right)+\left(\partial_{y}^{2} u_{x}\right)+\left(\partial_{z}^{2} u_{x}\right)+\left(\partial_{x} \partial_{y} u_{y}\right)\right. \\
\left.+\left(\partial_{x} \partial_{z} u_{z}\right)\right\} \\
-c_{2}\left\{\left(\partial_{x}^{2} u_{x}\right)+\left(\partial_{x} \partial_{y} u_{y}\right)+\left(\partial_{x} \partial_{z} u_{z}\right)\right\} \\
-\frac{1}{2} D_{1}\left\{\left(\partial_{y} n_{y}\right)+\frac{1}{2}\left[\left(\partial_{y}^{2} u_{x}\right)-\left(\partial_{x} \partial_{y} u_{y}\right)\right]\right. \\
\left.+\left(\partial_{z} n_{z}\right)+\frac{1}{2}\left[\left(\partial_{z}^{2} u_{x}\right)-\left(\partial_{x} \partial_{z} u_{z}\right)\right]\right\} \\
-\frac{1}{2} D_{2}\left\{\left(\partial_{y} n_{y}\right)+\left(\partial_{y}^{2} u_{x}\right)+\left(\partial_{z} n_{z}\right)\right. \\
\left.+\left(\partial_{z}^{2} u_{x}\right)\right\},
\end{gathered}
$$$$
0 \equiv \frac{\delta \mathcal{F}}{\delta u_{y}} \equiv-c_{1}\left\{\left(\partial_{x}^{2} u_{y}\right)+2\left(\partial_{y}^{2} u_{y}\right)+\left(\partial_{z}^{2} u_{y}\right)+\left(\partial_{x} \partial_{y} u_{x}\right)\right.
$$$$
\left.+\left(\partial_{y} \partial_{z} u_{z}\right)\right\}
$$$$
-c_{2}\left\{\left(\partial_{x} \partial_{y} u_{x}\right)+\left(\partial_{y}^{2} u_{y}\right)+\left(\partial_{y} \partial_{z} u_{z}\right)\right\}
$$$$
-\frac{1}{2} D_{1}\left\{-\left(\partial_{x} n_{y}\right)+\frac{1}{2}\left[\left(\partial_{x}^{2} u_{y}\right)-\left(\partial_{x} \partial_{y} u_{x}\right)\right]\right\}
$$$$
-\frac{1}{2} D_{2}\left\{\left(\partial_{x} n_{y}\right)-\left(\partial_{x}^{2} u_{y}\right)\right\}
$$$$
0 \equiv \frac{\delta \mathcal{F}}{\delta u_{z}} \equiv-c_{1}\left\{\left(\partial_{x}^{2} u_{z}\right)+\left(\partial_{y}^{2} u_{z}\right)+2\left(\partial_{z}^{2} u_{z}\right)+\left(\partial_{x} \partial_{z} u_{x}\right)\right.
$$$$
\left.+\left(\partial_{y} \partial_{z} u_{y}\right)\right\}
$$$$
-c_{2}\left\{\left(\partial_{x} \partial_{z} u_{x}\right)+\left(\partial_{y} \partial_{z} u_{y}\right)+\left(\partial_{z}^{2} u_{z}\right)\right\}
$$$$
-\frac{1}{2} D_{1}\left\{-\left(\partial_{x} n_{z}\right)+\frac{1}{2}\left[\left(\partial_{x}^{2} u_{z}\right)-\left(\partial_{x} \partial_{z} u_{x}\right)\right]\right\}
$$$$
-\frac{1}{2} D_{2}\left\{\left(\partial_{x} n_{z}\right)-\left(\partial_{x}^{2} u_{z}\right)\right\} \text {, }
$$$$
0 \equiv \frac{\delta \mathcal{F}}{\delta n_{y}} \equiv \frac{1}{2} D_{1}\left\{2 n_{y}-\left(\partial_{x} u_{y}\right)+\left(\partial_{y} u_{x}\right)\right\}
$$$$
+\frac{1}{2} D_{2}\left\{\left(\partial_{y} u_{x}\right)+\left(\partial_{x} u_{y}\right)\right\}
$$$$
-\epsilon_{a} E^{2} n_{y}
$$$$
-K_{1}\left\{\left(\partial_{y}^{2} n_{y}\right)+\left(\partial_{y} \partial_{z} n_{z}\right)\right\}
$$$$
-K_{2}\left\{-\left(\partial_{y} \partial_{z} n_{z}\right)+\left(\partial_{z}^{2} n_{y}\right)\right\}
$$$$
-K_{3}\left(\partial_{x}^{2} n_{y}\right)
$$

$$
\begin{aligned}
0 \equiv \frac{\delta \mathcal{F}}{\delta n_{z}} \equiv & \frac{1}{2} D_{1}\left\{2 n_{z}-\left(\partial_{x} u_{z}\right)+\left(\partial_{z} u_{x}\right)\right\} \\
& +\frac{1}{2} D_{2}\left\{\left(\partial_{z} u_{x}\right)+\left(\partial_{x} u_{z}\right)\right\} \\
& -K_{1}\left\{\left(\partial_{y} \partial_{z} n_{y}\right)+\left(\partial_{z}^{2} n_{z}\right)\right\} \\
& -K_{2}\left\{-\left(\partial_{y} \partial_{z} n_{y}\right)+\left(\partial_{y}^{2} n_{z}\right)\right\} \\
& -K_{3}\left(\partial_{x}^{2} n_{z}\right) .
\end{aligned}
$$

\section{Appendix C. Minima of the critical electric field amplitude in the twist geometry}

In the following appendix we will investigate expressions (35)-(37) in order to characterize the possible threshold instabilities in the twist geometry. An analysis of the Hessian will demonstrate that in this geometry the sample thickness is the decisive parameter for the quality of the instability.

Candidates for minima of $\epsilon_{a} E^{2}\left(q_{x}, q_{y}, q_{z}\right)$ in $\boldsymbol{q}$ space are obtained by setting the derivatives of expression (35) with respect to $q_{x}, q_{y}$, and $q_{z}$ equal to zero,

$$
\frac{\partial\left(\epsilon_{a} E^{2}\right)}{\partial q_{i}}\left(q_{x}, q_{y}, q_{z}\right)=2 q_{i} \frac{\partial\left(\epsilon_{a} E^{2}\right)}{\partial\left(q_{i}^{2}\right)}\left(q_{x}, q_{y}, q_{z}\right) \stackrel{!}{=} 0,
$$

where $i=x, y, z$. We note that the point $(0,0,0)$ in $\boldsymbol{q}$ space always satisfies these conditions, because the $q_{i}$ 's ( $i=x, y, z)$ enter $\epsilon_{a} E^{2}\left(q_{x}, q_{y}, q_{z}\right)$ only quadratically. This point corresponds to the trivial solution of vanishing amplitudes $A=B=N=0$ in equation (33). On the contrary, an instability is characterized by a nonvanishing wave vector $\boldsymbol{q}_{\boldsymbol{c}}=\left(q_{x, c}, q_{y, c}, q_{z, c}\right)$. In this case, the condition $q_{z, c} \neq 0$ must be satisfied because of equation (29). More precisely, we can set

$$
q_{z, c}= \pm \frac{\pi}{d}
$$

because of the following reasons. We introduce ansatz (30) into the expression for the generalized energy density $F$ in equation (B.1). Equations (31) and (32) are taken into account by $G=H=0$. From the boundary condition (29) it follows that $q_{z}=n \frac{\pi}{d}, n$ being an integer number. Consequently, we can easily integrate out the $z$-dependence of $F$, which is one step in determining the generalized energy $\mathcal{F}=\int_{V} F \mathrm{~d}^{3} r$. The result contains the wave number $q_{z}$ only as a factor $q_{z}^{2}$ of an expression which is positive due to the thermodynamic stability conditions listed at the end of Section 2. Therefore, $\mathcal{F}$ derived from equation (B.1) is the lower the lower is $\left|q_{z}\right|$, and for a nontrivial solution we obtain equation (C.2). Thus, if $(0,0,0)$ is the position of a global minimum of $\epsilon_{a} E^{2}\left(q_{x}, q_{y}, q_{z}\right)$ in $\boldsymbol{q}$ space, we conclude that $\boldsymbol{q}_{\boldsymbol{c}}$ is given by a point $\left(q_{x, c}, q_{y, c}, \pm \frac{\pi}{d}\right)$ with $q_{x, c}$ and $q_{y, c}$ close to zero. Further statements on this point clearly exceed the borders of our linear stability analysis. So, in general, we have to assume that in this case the 
instability is of class (I) and we may find undulations in both lateral directions $\hat{\boldsymbol{x}}$ and $\hat{\boldsymbol{y}}$ with critical wave numbers $q_{x, c}^{2}, q_{y, c}^{2} \ll q_{z, c}^{2}$.

In order to investigate whether we find a minimum of $\epsilon_{a} E^{2}\left(q_{x}, q_{y}, q_{z}\right)$ near the point $\left(0,0, \pm \frac{\pi}{d}\right)$, we inspect whether the Hessian is positive definite at this point, or equivalently, whether its eigenvalues are positive. The Hessian at $\left(0,0, \pm \frac{\pi}{d}\right)$ reads

$$
\left(\begin{array}{ccc}
2 K_{3}-\frac{2 \alpha^{2}}{c_{1}}\left(\frac{d}{\pi}\right)^{2} & 0 & 0 \\
0 & 2 K_{1}-\frac{2 \tilde{\alpha}^{2}}{\tilde{\tilde{\beta}}}\left(\frac{d}{\pi}\right)^{2} & 0 \\
0 & 0 & 2 K_{2}
\end{array}\right)
$$

and we infer that the sample thickness $d$ is the decisive parameter when a sample of a nematic SCLSCE with specific material parameters is given. We find a minimum close to $\left(0,0, \pm \frac{\pi}{d}\right)$ if the sample thickness satisfies the conditions

$$
\begin{aligned}
& d<d_{1}=\pi \frac{\sqrt{K_{1} \tilde{\beta}}}{|\tilde{\alpha}|} \text { and } \\
& d<d_{2}=\pi \frac{\sqrt{K_{3} c_{1}}}{|\alpha|} .
\end{aligned}
$$

$\left(c_{1}, \beta, \tilde{\beta}\right.$, and the Frank coefficients are positive due to the thermodynamic stability conditions.)

As the Hessian (C.3) is diagonal its elements correspond to its eigenvalues, and we can relate the first two of them to the curvatures of the surface $\epsilon_{a} E^{2}\left(q_{x}, q_{y}, \pm \frac{\pi}{d}\right)$ over the $\left(q_{x}, q_{y}\right)$-plane. Increasing the sample thickness, the point $(0,0)$ first turns into a saddle point and then into a point where the surface $\epsilon_{a} E^{2}\left(q_{x}, q_{y}, \pm \frac{\pi}{d}\right)$ over the $\left(q_{x}, q_{y}\right)$-plane has a local maximum.

We note that the limit

$$
\lim _{d \rightarrow \infty} \epsilon_{a} E^{2}\left(0,0, \pm \frac{\pi}{d}\right)=D_{1}
$$

would give the material parameter $D_{1}$ connected solely to the relative rotations. However, this limit is not helpful to design an experiment in order to determine $D_{1}$ : for sample thicknesses that are too large we do not find the critical point in $\boldsymbol{q}$ space close to $\left(0,0, \pm \frac{\pi}{d}\right)$.

The question we have to address now is whether there exist other three-dimensional minima of class (I). We could show by analytic calculation that in the limit of $d \rightarrow \infty$, or equivalently $q_{z, c} \rightarrow 0$, no such other minima exist, because $q_{x}$ or $q_{y}$ would become imaginary in this case. For finite $d$ we derived an implicit three-dimensional solution under special circumstances, however, we do not attribute physical relevance to this solution. Furthermore, various plots of $\epsilon_{a} E^{2}\left(q_{x}, q_{y}, \pm \frac{\pi}{d}\right)$ for different values of the material parameters did not reveal any evidence of a minimum which is characterized simultaneously by $q_{x} \neq 0$ and $q_{y} \neq 0$. For all these reasons we conclude that there is no relevant minimum of class (I) existing, except for the one close to the point $\left(0,0, \pm \frac{\pi}{d}\right)$ which we discussed above by the Hessian (C.3) and which might be of three-dimensional character.
We thus only have to look for two-dimensional candidates that may minimize $\epsilon_{a} E^{2}\left(q_{x}, q_{y}, q_{z}\right)$. As discussed before, we set $q_{z, c}= \pm \frac{\pi}{d}$. In the case of $q_{x}=0$ we obtain two candidates for a minimum, namely the point $\boldsymbol{q}=\left(0,0, \pm \frac{\pi}{d}\right)$ as before, and additionally, as a candidate for a minimum of class (II),

$$
\boldsymbol{q}^{(\mathrm{II})}=\left(0, \pm \sqrt{\frac{\pi}{d}\left(\frac{|\tilde{\alpha}|}{\sqrt{K_{1} \tilde{\beta}}}-\frac{\pi}{d}\right)}, \pm \frac{\pi}{d}\right)
$$

The latter solution only exists for a sample thickness $d$ that does not satisfy inequality (C.4). In this case, the evaluation of the two-dimensional Hessian shows that we obtain a minimum at $\boldsymbol{q}^{(\mathrm{II})}$ in the plane $q_{x}=0$. For an "="-sign in condition (C.4), the two solutions $\boldsymbol{q}^{(\mathrm{II})}$ and $\boldsymbol{q}=\left(0,0, \pm \frac{\pi}{d}\right)$ coincide. The absolute value of the wave number $q_{y}^{(\mathrm{II})}$ becomes largest at a sample thickness

$$
d_{\max }^{(\mathrm{II})}=\frac{2 \pi}{|\tilde{\alpha}|} \sqrt{K_{1} \tilde{\beta}} .
$$

For $q_{y}=0$ we analogously derive two candidates for a minimum, which are the point $\boldsymbol{q}=\left(0,0, \pm \frac{\pi}{d}\right)$ again, as well as a candidate for a minimum of class (III),

$$
\boldsymbol{q}^{(\mathrm{III})}=\left( \pm \sqrt{\frac{\pi}{d} \frac{c_{1}}{\beta}\left(\frac{|\alpha|}{\sqrt{K_{3} c_{1}}}-\frac{\pi}{d}\right)}, 0, \pm \frac{\pi}{d}\right)
$$

Now, the second solution only exists if the sample thickness $d$ violates inequality (C.5). Then, we obtain a minimum at $\boldsymbol{q}^{(\mathrm{III})}$ in the plane $q_{y}=0$, which can be inferred from the two dimensional Hessian. The two solutions $\boldsymbol{q}^{(\mathrm{III})}$ and $\boldsymbol{q}=\left(0,0, \pm \frac{\pi}{d}\right)$ coincide for an "="-sign in condition (C.5), and $\left|q_{x}^{(\mathrm{III})}\right|$ becomes largest for

$$
d_{\max }^{(\mathrm{III})}=\frac{2 \pi}{|\alpha|} \sqrt{K_{3} c_{1}} .
$$

\section{Appendix D. First term dominating in inequality (42)}

For completeness we want to have a look at the situation that occurs when the first term dominates in inequality (42). This is the case when either $D_{2} \rightarrow 0$ or $D_{2} \rightarrow \pm 2 \sqrt{c_{1} D_{1}}$. Condition (42) can then be rewritten as

$$
\frac{K_{3}}{K_{1}}>1+\frac{D_{1}-2 D_{2}}{4 c_{1}} .
$$

Consequently, now the ratio of the Frank coefficients determines the quality of the instability at onset. For $D_{2} \rightarrow$ 0 , we expect splay and twist undulations with $q_{x}=0$ if $\frac{K_{3}}{K_{1}}>1+\frac{D_{1}}{4 c_{1}}$, otherwise bend and twist undulations with $q_{y}=0$. For $D_{2} \rightarrow \pm 2 \sqrt{c_{1} D_{1}}$, splay and twist undulations with $q_{x}=0$ are predicted if $\frac{K_{3}}{K_{1}}>\left(1 \mp \frac{1}{2} \sqrt{\frac{D_{1}}{c_{1}}}\right)^{2}$, and again bend and twist undulations with $q_{y}=0$ otherwise. 
We also investigated what happens in our example of assigning numerical values to the material parameters. For this purpose we chose $D_{2}=0, D_{2}=+2 \sqrt{c_{1} D_{1}}$, and $D_{2}=-2 \sqrt{c_{1} D_{1}}$, respectively. In each case we first took the numerical values for $K_{1}$ and $K_{3}$ as noted in Section 4 , and then in a second step switched the two values. Plotting the surface $\epsilon_{a} E^{2}\left(q_{x}, q_{y}, \pm \frac{\pi}{d}\right)$ for each of the resulting six cases we could verify the results described above.

\section{References}

1. H. Finkelmann, H.-J. Kock, G. Rehage, Makromol. Chem., Rapid Commun. 2, 317 (1981).

2. H. Finkelmann, H. Ringsdorf, J.H. Wendorff, Makromol. Chem. 179, 273 (1978).

3. J. Küpfer, H. Finkelmann, Makromol. Chem., Rapid Commun. 12, 717 (1991).

4. A. Komp, J. Rühe, H. Finkelmann, Macromol. Rapid Commun. 26, 813 (2005).

5. H.R. Brand, K. Kawasaki, Macromol. Rapid Commun. 15 251 (1994).

6. D.-U. Cho, Y. Yusuf, P.E. Cladis, H.R. Brand, H. Finkelmann, S. Kai, Jpn. J. Appl. Phys. 46, 1106 (2007).

7. A. Lebar, Z. Kutnjak, S. Žumer, H. Finkelmann, A. Sánchez-Ferrer, B. Zalar, Phys. Rev. Lett. 94, 197801 (2005).

8. M. Hébert, R. Kant, P.-G. de Gennes, J. Phys. I 7, 909 (1997).

9. K. Urayama, S. Honda, T. Takigawa, Macromolecules 38, 3574 (2005).
10. Y. Yusuf, J.H. Huh, P.E. Cladis, H.R. Brand, H. Finkelmann, S. Kai, Phys. Rev. E 71, 061702 (2005).

11. P.G. de Gennes, J. Prost, The Physics of Liquid Crystals (Clarendon Press, Oxford, 1993).

12. K. Urayama, S. Honda, T. Takigawa, Macromolecules 39, 1943 (2006).

13. C.-C. Chang, L.-C. Chien, R.B. Meyer, Phys. Rev. E 56, 595 (1997).

14. E.M. Terentjev, M. Warner, P. Bladon, J. Phys. II 4, 667 (1994).

15. E.M. Terentjev, M. Warner, R.B. Meyer, J. Yamamoto, Phys. Rev. E 60, 1872 (1999).

16. G. Skačej, C. Zannoni, Eur. Phys. J. E 20, 289 (2006).

17. O. Müller, H.R. Brand, Eur. Phys. J. E 17, 53 (2005).

18. A.M. Menzel, H.R. Brand, Phys. Rev. E 75, 011707 (2007).

19. H.R. Brand, H. Pleiner, Physica A 208, 359 (1994).

20. A.M. Menzel, H.R. Brand, J. Chem. Phys. 125, 194704 (2006).

21. J. Küpfer, E. Nishikawa, H. Finkelmann, Polym. Adv. Techn. 5, 110 (1994).

22. L.D. Landau, E.M. Lifschitz, Elasticity Theory (Pergamon Press, New York, 1959).

23. P.G. de Gennes, in Liquid Crystals of One- and TwoDimensional Order, edited by W. Helfrich, G. Heppke (Springer, Berlin, 1980) pp. $231 \mathrm{ff}$.

24. H.R. Brand, H. Pleiner, P. Martinoty, Soft Matter 2, 182 (2006).

25. A.M. Menzel, H. Pleiner, H.R. Brand, J. Chem. Phys. 126, 234901 (2007).

26. F. Lonberg, R.B. Meyer, Phys. Rev. Lett. 55, 718 (1985).

27. N. Schwenk, H.W. Spiess, J. Phys. II 3, 865 (1993).

28. G. Cordoyiannis, A. Lebar, B. Zalar, S. Žumer, H. Finkelmann, Z. Kutnjak, Phys. Rev. Lett. 99, 197801 (2007). 